\title{
Characterisation of Exponential Convergence to Nonequilibrium Limits for Stochastic Volterra Equations
}

\author{
John A. D. Appleby, ${ }^{1}$ Siobhán Devin, ${ }^{2}$ and David W. Reynolds ${ }^{1}$ \\ ${ }^{1}$ School of Mathematical Sciences, Dublin City University, Dublin 9, Ireland \\ 2 School of Mathematical Sciences, University College Cork, Cork, Ireland \\ Correspondence should be addressed to John A. D. Appleby, john.appleby@dcu.ie \\ Received 25 October 2007; Accepted 11 May 2008 \\ Recommended by Jiongmin Yong
}

This paper considers necessary and sufficient conditions for the solution of a stochastically and deterministically perturbed Volterra equation to converge exponentially to a nonequilibrium and nontrivial limit. Convergence in an almost sure and $p$ th mean sense is obtained.

Copyright (C) 2008 John A. D. Appleby et al. This is an open access article distributed under the Creative Commons Attribution License, which permits unrestricted use, distribution, and reproduction in any medium, provided the original work is properly cited.

\section{Introduction}

In this paper, we study the exponential convergence of the solution of

$$
\begin{gathered}
d X(t)=\left(A X(t)+\int_{0}^{t} K(t-s) X(s) d s+f(t)\right) d t+\Sigma(t) d B(t), \quad t>0, \\
X(0)=X_{0}
\end{gathered}
$$

to a nontrivial random variable. Here the solution $X$ is an $n$-dimensional vector-valued function on $[0, \infty), A$ is a real $n \times n$-dimensional matrix, $K$ is a continuous and integrable $n \times n$-dimensional matrix-valued function on $[0, \infty), f$ is a continuous $n$-dimensional vectorvalued function on $[0, \infty), \Sigma$ is a continuous $n \times d$-dimensional matrix-valued function on $[0, \infty)$ and $B(t)=\left(B_{1}(t), B_{2}(t), \ldots, B_{d}(t)\right)$, where each component of the Brownian motion is independent. The initial condition $X_{0}$ is a deterministic constant vector.

The solution of (1.1a)-(1.1b) can be written in terms of the solution of the resolvent equation

$$
\begin{gathered}
R^{\prime}(t)=A R(t)+\int_{0}^{t} K(t-s) R(s) d s, \quad t>0, \\
R(0)=I,
\end{gathered}
$$


where the matrix-valued function $R$ is known as the resolvent or fundamental solution. In [1], the authors studied the asymptotic convergence of the solution $R$ of (1.2a)-(1.2b) to a nontrivial limit $R_{\infty}$. It was found that $R-R_{\infty}$ being integrable and the kernel being exponentially integrable were necessary and sufficient for exponential convergence. This built upon a result of Murakami [2] who considered the exponential convergence of the solution to a trivial limit and a result of Krisztin and Terjéki [3] who obtained necessary and sufficient conditions for the integrability of $R-R_{\infty}$. A deterministically perturbed version of $(1.2 \mathrm{a})-(1.2 \mathrm{~b})$,

$$
\begin{gathered}
x^{\prime}(t)=A x(t)+\int_{0}^{t} K(t-s) x(s) d s+f(t), \quad t>0, \\
x(0)=x_{0},
\end{gathered}
$$

was also studied in [1]. It was shown that the exponential decay of the tail of the perturbation $f$ combined with the integrability of $R-R_{\infty}$ and the exponential integrability of the kernel were necessary and sufficient conditions for convergence to a nontrivial limit.

The case where $(1.2 \mathrm{a})-(1.2 \mathrm{~b})$ is stochastically perturbed

$$
\begin{gathered}
d X(t)=\left(A X(t)+\int_{0}^{t} K(t-s) X(s) d s\right) d t+\Sigma(t) d B(t), \quad t>0, \\
X(0)=X_{0},
\end{gathered}
$$

has been considered. Various authors including Appleby and Freeman [4], Appleby and Riedle [5], Mao [6], and Mao and Riedle [7] have studied convergence to equilibrium. In particular the paper by Appleby and Freeman [4] considered the speed of convergence of solutions of (1.4a)-(1.4b) to equilibrium. It was shown that under the condition that the kernel does not change sign on $[0, \infty)$ then (i) the almost sure exponential convergence of the solution to zero, (ii) the $p$ th mean exponential convergence of the solution to zero, and (iii) the exponential integrability of the kernel and the exponential square integrability of the noise are equivalent.

Two papers by Appleby et al. [8,9] considered the convergence of solutions of (1.4a)(1.4b) to a nonequilibrium limit in the mean square and almost sure senses, respectively. Conditions on the resolvent, kernel, and noise for the convergence of solutions to an explicit limiting random variable were found. A natural progression from this work is the analysis of the speed of convergence.

This paper examines (1.1a)-(1.1b) and builds on the results in $[1,8,9]$. The analysis of (1.1a)-(1.1b) is complicated, particularly in the almost sure case, due to presence of both a deterministic and stochastic perturbation. Nonetheless, the set of conditions which characterise the exponential convergence of the solution of (1.1a)-(1.1b) to a nontrivial random variable is found. It can be shown that the integrability of $R-R_{\infty}$, the exponential integrability of the kernel, the exponential square integrability of the noise combined with the exponential decay of the tail of the deterministic perturbation, $t \mapsto \int_{t}^{\infty} f(s) d s$, are necessary and sufficient conditions for exponential convergence of the solution to a nontrivial random limit.

\section{Mathematical preliminaries}

In this section, we introduce some standard notation as well as giving a precise definition of (1.1a)-(1.1b) and its solution. 
Let $\mathbb{R}$ denote the set of real numbers and let $\mathbb{R}^{n}$ denote the set of $n$-dimensional vectors with entries in $\mathbb{R}$. Denote by $\mathbf{e}_{i}$ the $i$ th standard basis vector in $\mathbb{R}^{n}$. Denote by $\|A\|$ the standard Euclidean norm for a vector $A=\left(a_{1}, \ldots, a_{n}\right)$ given by

$$
\|A\|^{2}=\sum_{i=1}^{n} a_{i}^{2}=\operatorname{tr} A A^{T},
$$

where tr denotes the trace of a square matrix.

Let $\mathbb{R}^{n \times n}$ be the space of $n \times n$ matrices with real entries where $I$ is the identity matrix. Let $\operatorname{diag}\left(a_{1}, a_{2}, \ldots, a_{n}\right)$ denote the $n \times n$ matrix with the scalar entries $a_{1}, a_{2}, \ldots, a_{n}$ on the diagonal and 0 elsewhere. For $A=\left(a_{i j}\right) \in \mathbb{R}^{n \times d}$ the norm denoted by $\|\cdot\|$ is defined by

$$
\|A\|^{2}=\sum_{i=1}^{n} \sum_{j=1}^{d}\left|a_{i j}\right|^{2}
$$

The set of complex numbers is denoted by $\mathbb{C}$; the real part of $z$ in $\mathbb{C}$ being denoted by $\operatorname{Re} z$. The Laplace transform of the function $A:[0, \infty) \rightarrow \mathbb{R}^{n \times d}$ is defined as

$$
\widehat{A}(z)=\int_{0}^{\infty} A(t) e^{-z t} d t
$$

If $\epsilon \in \mathbb{R}$ and $\int_{0}^{\infty}\|A(t)\| e^{-\epsilon t} d t<\infty$ then $\widehat{A}(z)$ exists for $\operatorname{Re} z \geq \epsilon$ and $z \mapsto \widehat{A}(z)$ is analytic for $\operatorname{Re} z>\epsilon$.

If $J$ is an interval in $\mathbb{R}$ and $V$ a finite-dimensional normed space with norm $\|\cdot\|$ then $C(J, V)$ denotes the family of continuous functions $\phi: J \rightarrow V$. The space of Lebesgue integrable functions $\phi:[0, \infty) \rightarrow V$ will be denoted by $L^{1}([0, \infty), V)$ where $\int_{0}^{\infty}\|\phi(t)\| d t<\infty$. The space of Lebesgue square-integrable functions $\phi:[0, \infty) \rightarrow V$ will be denoted by $L^{2}([0, \infty), V)$ where $\int_{0}^{\infty}\|\phi(t)\|^{2} d t<\infty$. When $V$ is clear from the context, it is omitted it from the notation. satisfies

We now make our problem precise. We assume that the function $K:[0, \infty) \rightarrow \mathbb{R}^{n \times n}$

$$
K \in C\left([0, \infty), \mathbb{R}^{n \times n}\right) \cap L^{1}\left([0, \infty), \mathbb{R}^{n \times n}\right),
$$

the function $f:[0, \infty) \rightarrow \mathbb{R}^{n}$ satisfies

$$
f \in C\left([0, \infty), \mathbb{R}^{n}\right) \cap L^{1}\left([0, \infty), \mathbb{R}^{n}\right),
$$

and the function $\Sigma:[0, \infty) \rightarrow \mathbb{R}^{n \times d}$ satisfies

$$
\Sigma \in C\left([0, \infty), \mathbb{R}^{n \times d}\right) .
$$

Due to (2.4) we may define $K_{1}$ to be a function $K_{1} \in C\left([0, \infty), \mathbb{R}^{n \times n}\right)$ with

$$
K_{1}(t)=\int_{t}^{\infty} K(s) d s, \quad t \geq 0,
$$

where this function defines the tail of the kernel $K$. Similarly, due to (2.5), we may define $f_{1}$ to be a function $f_{1} \in C\left([0, \infty), \mathbb{R}^{n}\right)$ given by

$$
f_{1}(t)=\int_{t}^{\infty} f(s) d s, \quad t \geq 0
$$


We let $\{B(t)\}_{t \geq 0}$ denote $d$-dimensional Brownian motion on a complete probability space $\left(\Omega, \mathcal{F},\left\{\mathscr{F}^{B}(t)\right\}_{t \geq 0}, \mathbb{P}\right)$ where the filtration is the natural one $\mathcal{F}^{B}(t)=\sigma\{B(s): 0 \leq s \leq t\}$.

Under the hypothesis (2.4), it is well known that (1.2a)-(1.2b) has a unique continuous solution $R$, which is continuously differentiable. We define the function $t \mapsto X\left(t ; X_{0}, f, \Sigma\right)$ to be the unique solution of the initial value problem (1.1a)-(1.1b). If $\Sigma$ and $f$ are continuous then for any deterministic initial condition $X_{0}$ there exists an almost surely unique continuous and $\mathcal{F}^{B}$-adapted solution to (1.1a)-(1.1b) given by

$$
X\left(t ; X_{0}, \Sigma, f\right)=R(t) X_{0}+\int_{0}^{t} R(t-s) f(s) d s+\int_{0}^{t} R(t-s) \Sigma(s) d B(s), \quad t \geq 0 .
$$

When $X_{0}, f$, and $\Sigma$ are clear from the context, we omit them from the notation.

The notion of convergence and integrability in $p$ th mean and almost sure senses are now defined: the $\mathbb{R}^{n}$-valued stochastic process $\{X(t)\}_{t \geq 0}$ converges in pth mean to $X_{\infty}$ if $\lim _{t \rightarrow \infty} \mathbb{E}\left\|X(t)-X_{\infty}\right\|^{p}=0$; the process is pth mean exponentially convergent to $X_{\infty}$ if there exists a deterministic $\beta_{p}>0$ such that

$$
\limsup _{t \rightarrow \infty} \frac{1}{t} \log \left(\mathbb{E}\left\|X(t)-X_{\infty}\right\|^{p}\right) \leq-\beta_{p}
$$

we say that the difference between the stochastic process $\{X(t)\}_{t \geq 0}$ and $X_{\infty}$ is integrable in the pth mean sense if

$$
\int_{0}^{\infty} \mathbb{E}\left\|X(t)-X_{\infty}\right\|^{p} d t<\infty
$$

If there exists a P-null set $\Omega_{0}$ such that for every $\omega \notin \Omega_{0}$, the following holds: $\lim _{t \rightarrow \infty} X(t, \omega)=$ $X_{\infty}(\omega)$, then $X$ converges almost surely to $X_{\infty}$; we say $X$ is almost surely exponentially convergent to $X_{\infty}$ if there exists a deterministic $\beta_{0}>0$ such that

$$
\limsup _{t \rightarrow \infty} \frac{1}{t} \log \left\|X(t, \omega)-X_{\infty}(\omega)\right\| \leq-\beta_{0} \text {, a.s. }
$$

Finally, the difference between the stochastic process $\{X(t)\}_{t \geq 0}$ and $X_{\infty}$ is square integrable in the almost sure sense if

$$
\int_{0}^{\infty}\left\|X(t, \omega)-X_{\infty}(\omega)\right\|^{2} d t<\infty
$$

Henceforth, $\mathbb{E}\left[X^{p}\right]$ will be denoted by $\mathbb{E} X^{p}$ except in cases where the meaning may be ambiguous. A number of inequalities are used repeatedly in the sequel; they are stated here for clarity. If, for $p, q \in(0, \infty)$, the finite-dimensional random variables $X$ and $Y$ satisfy $\mathbb{E}\|X\|^{p}<\infty$ and $\mathbb{E}\|Y\|^{q}<\infty$, respectively, then the Lyapunov inequality is useful when considering the $p$ th mean behaviour of random variables as any exponent $p>0$ may be considered:

$$
\mathbb{E}\left[\|X\|^{p}\right]^{1 / p} \leq \mathbb{E}\left[\|X\|^{q}\right]^{1 / q}, \quad 0<p \leq q .
$$

The following proves useful in manipulating norms:

$$
\left(\sum_{i=1}^{n}\left|x_{i}\right|\right)^{k} \leq n^{k-1} \sum_{i=1}^{n}\left|x_{i}\right|^{k}, \quad n, k \in \mathbb{N} .
$$




\section{Discussion of results}

We begin by stating the main result of this paper. That is, we state the necessary and sufficient conditions required on the resolvent, kernel, deterministic perturbation, and noise terms for the solution of (1.1a)-(1.1b) to converge exponentially to a limiting random variable. In this paper, we are particularly interested in the case when the limiting random variable is nontrivial, although the result is still true for the case when the limiting value is zero.

Theorem 3.1. Let $K$ satisfy (2.4) and

$$
\int_{0}^{\infty} t^{2}\|K(t)\| d t<\infty
$$

let $\Sigma$ satisfy (2.6), and let $f$ satisfy (2.5). If $K$ satisfies

$$
\text { each entry of } K \text { does not change sign on }[0, \infty) \text {, }
$$

then the following are equivalent.

(i) There exists a constant matrix $R_{\infty}$ such that the solution $R$ of (1.2a)-(1.2b) satisfies

$$
R-R_{\infty} \in L^{2}\left([0, \infty), \mathbb{R}^{n \times n}\right),
$$

and there exist constants $\alpha>0, \gamma>0, \rho>0$, and $c_{1}>0$ such that $K$ satisfies

$$
\int_{0}^{\infty}\|K(s)\| e^{\alpha s} d s<\infty
$$

$\Sigma$ satisfies

$$
\int_{0}^{\infty}\|\Sigma(s)\|^{2} e^{2 \gamma s} d s<\infty
$$

and $f_{1}$, the tail of $f$, defined by (2.8) satisfies

$$
\left\|f_{1}(t)\right\| \leq c_{1} e^{-\gamma t}, \quad t \geq 0 .
$$

(ii) For all initial conditions $X_{0}$ and constants $p>0$ there exists an a.s. finite $\mathcal{F}^{B}(\infty)$ measurable random variable $X_{\infty}\left(X_{0}, \Sigma, f\right)$ with $\mathbb{E}\left\|X_{\infty}\right\|^{p}<\infty$ such that the unique continuous adapted process $X\left(\cdot ; X_{0}, \Sigma, f\right)$ which obeys (1.1a)-(1.1b) satisfies

$$
\mathbb{E}\left[\left\|X(t)-X_{\infty}\right\|^{p}\right] \leq m_{p}^{*} e^{-\beta_{p}^{*} t}, \quad t \geq 0,
$$

where $\beta_{p}^{*}$ and $m_{p}^{*}=m_{p}^{*}\left(X_{0}\right)$ are positive constants.

(iii) For all initial conditions $X_{0}$ there exists an a.s. finite $\mathcal{F}^{B}(\infty)$-measurable random variable $X_{\infty}\left(X_{0}, \Sigma, f\right)$ such that the unique continuous adapted process $X\left(\cdot ; X_{0}, \Sigma, f\right)$ which obeys (1.1a)-(1.1b) satisfies

$$
\limsup _{t \rightarrow \infty} \frac{1}{t} \log \left\|X(t)-X_{\infty}\right\| \leq-\beta_{0}^{*} \text { a.s., }
$$

where $\beta_{0}^{*}$ is a positive constant. 
The proof of Theorem 3.1 is complicated by the presence of two perturbations so as an initial step the case when $f:=0$ is considered. That is we consider the conditions required for exponential convergence of $(1.4 \mathrm{a})-(1.4 \mathrm{~b})$ to a limiting random variable.

Theorem 3.2. Let $K$ satisfy (2.4) and (3.1) and let $\Sigma$ satisfy (2.6). If $K$ satisfies (3.2) then the following are equivalent.

(i) There exists a constant matrix $R_{\infty}$ such that the solution $R$ of (1.2a)-(1.2b) satisfies (3.3) and there exist constants $\alpha>0$ and $\gamma>0$ such that $K$ and $\Sigma$ satisfy (3.4) and (3.5), respectively.

(ii) For all initial conditions $X_{0}$ and constants $p>0$ there exists an a.s. finite $\Psi^{B}(\infty)$ measurable random variable $X_{\infty}\left(X_{0}, \Sigma\right)$ with $\mathbb{E}\left\|X_{\infty}\right\|^{p}<\infty$ such that the unique continuous adapted process $X\left(\cdot ; X_{0}, \Sigma\right)$ which obeys $(1.4 \mathrm{a})-(1.4 \mathrm{~b})$ satisfies

$$
\mathbb{E}\left[\left\|X(t)-X_{\infty}\right\|^{p}\right] \leq m_{p} e^{-\beta_{p} t}, \quad t \geq 0,
$$

where $\beta_{p}$ and $m_{p}=m_{p}\left(X_{0}\right)$ are positive constants.

(iii) For all initial conditions $X_{0}$ there exists an a.s. finite $\Psi^{B}(\infty)$-measurable random variable $X_{\infty}\left(X_{0}, \Sigma\right)$ such that the unique continuous adapted process $X\left(\cdot ; X_{0}, \Sigma\right)$ which obeys (1.4a)-(1.4b) satisfies

$$
\limsup _{t \rightarrow \infty} \frac{1}{t} \log \left\|X(t)-X_{\infty}\right\| \leq-\beta_{0} \text { a.s., }
$$

where $\beta_{0}$ is a positive constant.

This result is interesting in its own right as it generalises a result in [4] where necessary and sufficient conditions for exponential convergence to zero are found. Theorem 3.2 collapses to this case if $R_{\infty}=0$.

It is interesting to note the relationship between the behaviour of the solutions of (1.1a)-(1.1b), (1.2a)-(1.2b), (1.3a)-(1.3b), and (1.4a)-(1.4b) and the behaviour of the inputs $K, f$, and $\Sigma$. It is seen in [1] that $K$ being exponentially integrable is the crucial condition for exponential convergence when we consider the resolvent equation. Each perturbed equation then builds on this resolvent case: for the deterministically perturbed equation we require the exponential integrability of $K$ and the exponential decay of the tail of the perturbation $f$ (see [1]); for the stochastically perturbed case we require the exponential integrability of $K$ and the exponential square integrability of $\Sigma$. In the stochastically and deterministically perturbed case it is seen that the perturbations do not interact in a way that exacerbates or diminishes the influence of the perturbations on the system: we can isolate the behaviours of the perturbations and show that the same conditions on the perturbations are still necessary and sufficient.

Theorem 3.1 has application in the analysis of initial history problems. In particular this theoretical result could be used to interpret the equation as an epidemiological model. Conditions under which a disease becomes endemic (which is the interpretation that is given when solutions settle down to a nontrivial limit) were studied in [9]. The theoretical results obtained in this paper could be exploited to highlight the speed at which this can occur within a population.

The remainder of this paper deals with the proofs of Theorems 3.1 and 3.2. In Section 4 we prove the sufficiency of conditions on $R, K$, and $\Sigma$ for the exponential convergence of 
John A. D. Appleby et al.

the solution of (1.4a)-(1.4b) while in Section 5 we prove the necessity of these conditions. In Section 6 we prove the sufficiency of conditions on $R, K, \Sigma$, and $f$ for the exponential convergence of the solution of (1.1a)-(1.1b), while Section 7 deals with the necessity of the condition on $\Sigma$. In Section 8 we combine our results to prove the main theorems, namely, Theorems 3.1 and 3.2.

\section{Sufficient conditions for exponential convergence of solutions of $(1.4 a)-(1.4 b)$}

In this section, sufficient conditions for exponential convergence of solutions of (1.4a)-(1.4b) to a nontrivial limit are obtained. Proposition 4.1 concerns convergence in the $p$ th mean sense while Proposition 4.2 deals with the almost sure case.

Proposition 4.1. Let $K$ satisfy (2.4) and (3.1), let $\Sigma$ satisfy (2.6) and $R_{\infty}$ be a constant matrix such that the solution $R$ of (1.2a)-(1.2b) satisfies (3.3). If there exist constants $\alpha>0$ and $\gamma>0$ such that (3.4) and (3.5) hold, then there exist constants $\beta_{p}>0$, independent of $X_{0}$, and $m_{p}=m_{p}\left(X_{0}\right)>0$, such that statement (ii) of Theorem 3.2 holds.

Proposition 4.2. Let $K$ satisfy (2.4) and (3.1), let $\Sigma$ satisfy (2.6) and $R_{\infty}$ be a constant matrix such that the solution $R$ of (1.2a)-(1.2b) satisfies (3.3). If there exist constants $\alpha>0$ and $\gamma>0$ such that (3.4) and (3.5) hold, then there exists a constant $\beta_{0}>0$, independent of $X_{0}$ such that statement (iii) of Theorem 3.2 holds.

In [8], the conditions which give mean square convergence to a nontrivial limit were considered. So a natural progression in this paper is the examination of the speed of convergence in the mean square case. Lemma 4.3 examines the case when $p=2$ in order to highlight this important case. This lemma may be then used when generalising the result to all $p>0$.

Lemma 4.3. Let $K$ satisfy (2.4) and (3.1), let $\Sigma$ satisfy (2.6), and let $R_{\infty}$ be a constant matrix such that the solution $R$ of (1.2a)-(1.2b) satisfies (3.3). If there exist constants $\alpha>0$ and $\gamma>0$ such that (3.4) and (3.5) hold, then there exist constants $\lambda>0$, independent of $X_{0}$, and $m=m\left(X_{0}\right)>0$, such that

$$
\mathbb{E}\left\|X(t)-X_{\infty}\right\|^{2} \leq m\left(X_{0}\right) e^{-2 \lambda t}, \quad t \geq 0 .
$$

From $[8,9]$ it is evident that $R-R_{\infty} \in L^{2}\left([0, \infty), \mathbb{R}^{n \times n}\right)$ is a more natural condition on the resolvent than $R-R_{\infty} \in L^{1}\left([0, \infty), \mathbb{R}^{n \times n}\right)$ when studying convergence of solutions of (1.4a)-(1.4b). However, the deterministic results obtained in [1] are based on the assumption that $R-R_{\infty} \in L^{1}\left([0, \infty), \mathbb{R}^{n \times n}\right)$. Lemma 4.4 is required in order to make use of these results in this paper; this result isolates conditions that ensure the integrability of $R-R_{\infty}$ once $R-R_{\infty}$ is square integrable.

Lemma 4.4. Let $K$ satisfy (2.4) and (3.1) and let $R_{\infty}$ be a constant matrix such that the solution $R$ of (1.2a)-(1.2b) satisfies (3.3). Then the solution $R$ of $(1.2 \mathrm{a})-(1.2 \mathrm{~b})$ satisfies

$$
R-R_{\infty} \in L^{1}\left([0, \infty), \mathbb{R}^{n \times n}\right) .
$$

We now state some supporting results. It is well known that the behaviour of the resolvent Volterra equation influences the behaviour of the perturbed equation. It is unsurprising therefore that an earlier result found in [1] concerning exponential convergence of the resolvent $R$ to a limit $R_{\infty}$ in needed in the proof of Theorems 3.1 and 3.2. 
Theorem 4.5. Let $K$ satisfy (2.4) and (3.1). Suppose there exists a constant matrix $R_{\infty}$ such that the solution $R$ of (1.2a)-(1.2b) satisfies (4.2). If there exists a constant $\alpha>0$ such that $K$ satisfies (3.4) then there exist constants $\beta>0$ and $c>0$ such that

$$
\left\|R(t)-R_{\infty}\right\| \leq c e^{-\beta t}, \quad t \geq 0
$$

In the proof of Propositions 4.1 and 4.2, an explicit representation of $X_{\infty}$ is required. In $[8,9]$ the asymptotic convergence of the solution of (1.4a)-(1.4b) was considered. Sufficient conditions for convergence were obtained and an explicit representation of $X_{\infty}$ was found:

Theorem 4.6. Let $K$ satisfy (2.4) and

$$
\int_{0}^{\infty} t\|K(t)\| d t<\infty
$$

and let $\Sigma$ satisfy (2.6) and

$$
\int_{0}^{\infty}\|\Sigma(t)\|^{2} d t<\infty
$$

Suppose that the resolvent $R$ of (1.2a)-(1.2b) satisfies (3.3). Then the solution $X$ of (1.4a)-(1.4b) satisfies $\lim _{t \rightarrow \infty} X(t)=X_{\infty}$ almost surely, where $X_{\infty}$ is an almost surely finite and $\mathcal{F}^{B}(\infty)$ measurable random variable given by

$$
X_{\infty}=R_{\infty}\left(X_{0}+\int_{0}^{\infty} \Sigma(t) d B(t)\right) \text { a.s. }
$$

Lemma 4.7 concerns the structure of $X_{\infty}$ in the almost sure case. It was proved in [9].

Lemma 4.7. Let $K$ satisfy (2.4) and (4.4). Suppose that for all initial conditions $X_{0}$ there is an almost surely finite random variable $X_{\infty}\left(X_{0}, \Sigma\right)$ such that the solution $t \mapsto X\left(t ; X_{0}, \Sigma\right)$ of $(1.4 \mathrm{a})-(1.4 \mathrm{~b})$ satisfies

$$
\begin{gathered}
\lim _{t \rightarrow \infty} X\left(t ; X_{0}, \Sigma\right)=X_{\infty}\left(X_{0}, \Sigma\right) \text { a.s., } \\
X\left(\cdot ; X_{0}, \Sigma\right)-X_{\infty}\left(X_{0}, \Sigma\right) \in L^{2}\left([0, \infty), \mathbb{R}^{n}\right) \text { a.s. }
\end{gathered}
$$

Then

$$
\left(A+\int_{0}^{\infty} K(s) d s\right) X_{\infty}=0 \text { a.s. }
$$

It is possible to apply this lemma using our a priori assumptions due to Theorem 4.8, which was proved in [9].

Theorem 4.8. Let $K$ satisfy (2.4) and (4.4) and let $\Sigma$ satisfy (2.6). If $\Sigma$ satisfies (4.5) and there exists a constant matrix $R_{\infty}$ such that the solution $R$ of (1.2a)-(1.2b) satisfies (3.3), then for all initial conditions $X_{0}$ there is an almost surely finite $\mathcal{F}^{B}(\infty)$-measurable random variable $X_{\infty}\left(X_{0}, \Sigma\right)$, such that the unique continuous adapted process $X\left(\cdot ; X_{0}, \Sigma\right)$ which obeys $(1.4 \mathrm{a})-(1.4 \mathrm{~b})$ satisfies (4.7).

Moreover, if the function $\Sigma$ also satisfies

$$
\int_{0}^{\infty} t\left\|R_{\infty} \Sigma(t)\right\|^{2} d t<\infty,
$$

then (4.8) holds. 
Lemma 4.9 below is required in the proof of Lemma 4.4. It is proved in [8]. Before citing this result some notation is introduced. Let $M=A+\int_{0}^{\infty} K(t) d t$ and $T$ be an invertible matrix such that $J=T^{-1} M T$ has Jordan canonical form. Let $e_{i}=1$ if all the elements of the $i$ th row of $J$ are zero, and $e_{i}=0$ otherwise. Let $D_{p}=\operatorname{diag}\left(e_{1}, e_{2}, \ldots, e_{n}\right)$ and put $P=T D_{p} T^{-1}$ and $Q=I-P$.

Lemma 4.9. Let $K$ satisfy (2.4) and (4.4). If there exists a constant matrix $R_{\infty}$ such that the resolvent $R$ of (1.2a)-(1.2b) satisfies (3.3), then

$$
\operatorname{det}[I+\widehat{F}(z)] \neq 0, \quad \operatorname{Re} z \geq 0,
$$

where $F$ is defined by

$$
F(t)=-e^{-t}(Q+Q A)-(e * Q K)(t)+P \int_{t}^{\infty} K(u) d u, \quad t \geq 0 .
$$

Lemma 4.10 concerns the moments of a normally distributed random variable. It can be extracted from [4, Theorem 3.3] and it is used in Proposition 4.1.

Lemma 4.10. Suppose the function $\sigma \in C\left([0, \infty) \times[0, \infty), \mathbb{R}^{p \times r}\right)$ then

$$
\mathbb{E}\left\|\int_{a}^{b} \sigma(s, t) d B(s)\right\|^{2 m} \leq d_{m}(p, r)\left(\int_{a}^{b}\|\sigma(s, t)\|^{2} d s\right)^{m},
$$

where $d_{m}(p, r)=p^{m+1} r^{2 m+1}(2 m) !\left(m ! 2^{m}\right)^{-1} c_{2}(p, r)^{m}$.

The following lemma is used in Proposition 4.2. A similar result is proved in [4].

Lemma 4.11. Suppose that $\widetilde{K} \in C\left([0, \infty), \mathbb{R}^{n \times n}\right) \cap L^{1}\left([0, \infty), \mathbb{R}^{n \times n}\right)$ and

$$
\int_{0}^{\infty}\|\tilde{K}(s)\| e^{\tilde{\alpha} s} d s<\infty .
$$

If $\tilde{\lambda}>0$ and $\tilde{\eta}=2 \tilde{\lambda} \wedge \tilde{\alpha}$ then

$$
\int_{0}^{t} e^{-2 \tilde{\lambda}(t-s)} e^{-\tilde{\alpha} s}\|\tilde{K}(s)\| d s \leq c e^{-\tilde{\eta} t}
$$

where $c$ is a positive constant.

The proofs of Propositions 4.1 and 4.2 and Lemmas 4.3 and 4.4 are now given.

Proof of Lemma 4.3. From Theorem 4.6 we see that $X(t) \rightarrow X_{\infty}$ almost surely where $X_{\infty}$ is given by (4.6), so we see that

$$
\mathbb{E}\left\|X_{\infty}\right\|^{2}=\mathbb{E}\left[\operatorname{tr}\left(X_{\infty} X_{\infty}^{T}\right)\right]=\left\|R_{\infty} X_{0}\right\|^{2}+\int_{0}^{\infty}\left\|R_{\infty} \Sigma(s)\right\|^{2} d s<\infty .
$$

Since

$$
\mathbb{E}\left[\left\|X(t)-X_{\infty}\right\|^{2}\right]=\mathbb{E}\left[\operatorname{tr}\left(X(t)-X_{\infty}\right)\left(X(t)-X_{\infty}\right)^{T}\right],
$$


we use (2.9) and (4.6) to expand the right hand side of (4.17) to obtain

$$
\mathbb{E}\left[\left\|X(t)-X_{\infty}\right\|^{2}\right]=\left\|\left(R(t)-R_{\infty}\right) X_{0}\right\|^{2}+\int_{0}^{t}\left\|\left(R(t-s)-R_{\infty}\right) \Sigma(s)\right\|^{2} d s+\int_{t}^{\infty}\left\|R_{\infty} \Sigma(s)\right\|^{2} d s .
$$

In order to obtain an exponential upper bound on (4.18) each term is considered individually. We begin by considering the first term on the right-hand side of (4.18). Using (3.1) and (3.3) we can apply Lemma 4.4 to obtain (4.2). Then using (3.1), (4.2), and (3.4) we see from Theorem 4.5 that

$$
\left\|\left(R(t)-R_{\infty}\right) X_{0}\right\|^{2} \leq c_{1}\left\|X_{0}\right\|^{2} e^{-2 \beta t} .
$$

We provide an argument to show that the second term decays exponentially. Using (3.5) and the fact that $R$ decays exponentially quickly to $R_{\infty}$ we can choose $0<\lambda<\min (\beta, \gamma)$ such that $e_{\lambda} \Sigma$ and $e_{\lambda}\left(R-R_{\infty}\right) \in L^{2}[0, \infty)$ where the function $e_{\lambda}$ is defined by $e_{\lambda}(t)=e^{\lambda t}$. Since the convolution of an $L^{2}[0, \infty)$ function with an $L^{2}[0, \infty)$ function is itself an $L^{2}[0, \infty)$ function we get

$$
e^{2 \lambda t} \int_{0}^{t}\left\|\left(R(t-s)-R_{\infty}\right) \Sigma(s)\right\|^{2} d s \leq \int_{0}^{t} e^{2 \lambda(t-s)}\left\|R(t-s)-R_{\infty}\right\|^{2} e^{2 \lambda s}\|\Sigma(s)\|^{2} d s \leq c_{2},
$$

and so the second term of (4.18) decays exponentially quickly.

We can show that the third term on the right hand side of (4.18) decays exponentially using (3.5) and the following argument:

$$
\bar{\Sigma}:=\int_{0}^{\infty}\|\Sigma(s)\|^{2} e^{2 \gamma s} d s \geq \int_{t}^{\infty}\|\Sigma(s)\|^{2} e^{2 \gamma s} d s \geq e^{2 \gamma t} \int_{t}^{\infty}\|\Sigma(s)\|^{2} d s .
$$

Combining these facts we see that

$$
\mathbb{E}\left[\left\|X(t)-X_{\infty}\right\|^{2}\right] \leq m\left(X_{0}\right) e^{-2 \lambda t},
$$

where $m\left(X_{0}\right)=c_{1}\left\|X_{0}\right\|^{2}+c_{2}+\bar{\Sigma}\left\|R_{\infty}\right\|^{2}$ and $\lambda<\min (\beta, \gamma)$.

Proof of Proposition 4.1. Consider the case where $0<p \leq 2$ and $p>2$ separately. We begin with the case where $0<p \leq 2$. The argument given by (4.16) shows that $\mathbb{E}\left[\left\|X_{\infty}\right\|^{2}\right]<\infty$. Now applying Lyapunov's inequality we see that

$$
\mathbb{E}\left\|X_{\infty}\right\|^{p} \leq \mathbb{E}\left[\left\|X_{\infty}\right\|^{2}\right]^{p / 2}<\infty .
$$

We now show that (3.9) holds for $0 \leq p \leq 2$. Lyapunov's inequality and Lemma 4.3 can be applied as follows:

$$
\mathbb{E}\left[\left\|X(t)-X_{\infty}\right\|^{p}\right] \leq \mathbb{E}\left[\left\|X(t)-X_{\infty}\right\|^{2}\right]^{p / 2} \leq m_{p}\left(X_{0}\right) e^{-\beta_{p} t}, \quad t \geq 0,
$$

where $m_{p}\left(X_{0}\right)=m\left(X_{0}\right)^{p / 2}$ and $\beta_{p}=\lambda p$.

Now consider the case where $p>2$. In this case, there exists a constant $m \in \mathbb{N}$ such that $2(m-1)<p \leq 2 m$. We now seek an upper bound on $\mathbb{E}\left\|X_{\infty}\right\|^{2 m}$ and $\mathbb{E}\left[\left\|X(t)-X_{\infty}\right\|^{2 m}\right]$, 
which will in turn give an upper bound on $\mathbb{E}\left\|X_{\infty}\right\|^{p}$ and $\mathbb{E}\left[\left\|X(t)-X_{\infty}\right\|^{p}\right]$ by using Lyapunov's inequality. By applying Lemma 4.10 we see that

$$
\mathbb{E}\left\|X_{\infty}\right\|^{2 m} \leq c\left\|R_{\infty} X_{0}\right\|^{2 m}+c\left(\int_{0}^{\infty}\left\|R_{\infty} \Sigma(s)\right\|^{2} d s\right)^{m}<\infty,
$$

where $c$ is a positive constant, so $\mathbb{E}\left\|X_{\infty}\right\|^{p} \leq \mathbb{E}\left[\left\|X_{\infty}\right\|^{2 m}\right]^{p / 2 m}<\infty$.

Now consider $\mathbb{E}\left[\left\|X(t)-X_{\infty}\right\|^{2 m}\right]$. Using the variation of parameters representation of the solution and the expression obtained for $X_{\infty}$, taking norms, raising both sides of the equation to the $2 m$ th power, then taking expectations across the inequality, we arrive at

$$
\begin{aligned}
\mathbb{E}\left[\left\|X(t)-X_{\infty}\right\|^{2 m}\right] & \\
\leq 3^{2 m-1}( & \left\|\left(R(t)-R_{\infty}\right) X_{0}\right\|^{2 m}+\mathbb{E}\left[\left\|\int_{0}^{t}\left(R(t-s)-R_{\infty}\right) \Sigma(s) d B(s)\right\|^{2 m}\right] \\
& \left.+\mathbb{E}\left[\left\|\int_{t}^{\infty} R_{\infty} \Sigma(s) d B(s)\right\|^{2 m}\right]\right) .
\end{aligned}
$$

We consider each term on the right hand side of (4.26). By Theorem 4.5 we have

$$
\left\|\left(R(t)-R_{\infty}\right) X_{0}\right\|^{2 m} \leq c_{1}\left\|X_{0}\right\|^{2 m} e^{-2 m \beta t} .
$$

Now, consider the second term on the right-hand side of (4.26). By (4.20) we see that $\int_{0}^{t} \|(R(t-$ $\left.s)-R_{\infty}\right) \Sigma(s) \|^{2} d s \leq c_{2} e^{-2 \lambda t}$ where $\lambda<\min (\beta, \gamma)$. Using this and Lemma 4.10 we see that

$$
\begin{aligned}
\mathbb{E}\left[\left\|\int_{0}^{t}\left(R(t-s)-R_{\infty}\right) \Sigma(s) d B(s)\right\|^{2 m}\right] & \leq d_{m}(n, d)\left(\int_{0}^{t}\left\|R(t-s)-R_{\infty}\right\|^{2}\|\Sigma(s)\|^{2} d s\right)^{m} \\
& \leq d_{m}(n, d) c_{2}^{m} e^{-2 m \lambda t} .
\end{aligned}
$$

Using (4.21) combined with Lemma 4.10 and Fatou's lemma, we show that the third term decays exponentially quickly:

$$
\begin{aligned}
\mathbb{E}\left[\left\|\int_{t}^{\infty} \Sigma(s) d B(s)\right\|^{2 m}\right] & \leq d_{m}(n, d)\left(\int_{t}^{\infty}\|\Sigma(s)\|^{2} d s\right)^{m} \\
& \leq d_{m}(n, d) \bar{\Sigma}^{m} e^{-2 m \gamma t} .
\end{aligned}
$$

Combining (4.27), (4.28), and (4.29) the inequality (4.26) becomes

$$
\begin{aligned}
& \mathbb{E}\left[\left\|X(t)-X_{\infty}\right\|^{2 m}\right] \\
& \quad \leq 3^{2 m-1}\left(c_{1}\left\|X_{0}\right\|^{2 m} e^{-2 m \beta t}+d_{m}(n, d) c_{2}^{m} e^{-2 m \lambda t}+d_{m}(n, d)\left\|R_{\infty}\right\|^{2 m} \bar{\Sigma}^{m} e^{-2 m \gamma t}\right) .
\end{aligned}
$$

Using Lyapunov's inequality, the inequality (4.30) implies

$$
\mathbb{E}\left[\left\|X(t)-X_{\infty}\right\|^{p}\right] \leq m_{p}\left(X_{0}\right) e^{-\beta_{p} t},
$$

where $m_{p}\left(X_{0}\right)=3^{p((2 m-1) / 2 m)}\left(c_{1}\left\|X_{0}\right\|^{2 m}+d_{m}(n, d) c_{2}^{m}+d_{m}(n, d)\left\|R_{\infty}\right\|^{2 m} \bar{\Sigma}^{m}\right)^{p / 2 m}$ and $\beta_{p}=\lambda p$. 
Proof of Proposition 4.2. In order to prove this proposition we show that

$$
\mathbb{E}\left[\sup _{n-1 \leq t \leq n}\left\|X(t)-X_{\infty}\right\|^{2}\right] \leq \tilde{m}\left(X_{0}\right) e^{-2 \eta(n-1)}, \quad \eta>0 .
$$

For each $t>0$ there exists $n \in \mathbb{N}$ such that $n-1 \leq t<n$. Define $\Delta(t)=X(t)-X_{\infty}$. Integrating (1.4a)-(1.4b) over $[n-1, t]$, then adding and subtracting $X_{\infty}$ on both sides we get

$$
\begin{aligned}
X(t)-X_{\infty}= & \left(X(n-1)-X_{\infty}\right)+\int_{n-1}^{t} A\left(X(s)-X_{\infty}\right) d s \\
& +\int_{n-1}^{t} \int_{0}^{s} K(s-u)\left(X(u)-X_{\infty}\right) d u d s+\int_{n-1}^{t} \Sigma(s) d B(s) \\
& +\int_{n-1}^{t}\left(A+\int_{0}^{\infty} K(u) d u\right) X_{\infty} d s-\int_{n-1}^{t} \int_{s}^{\infty} K(v) X_{\infty} d v d s .
\end{aligned}
$$

By applying Theorem 4.8, we see that (4.7) and (4.8) hold so Lemma 4.7 may be applied to obtain

$$
\Delta(t)=\Delta(n-1)+\int_{n-1}^{t}(A \Delta(s)+(K * \Delta)(s)) d s+\int_{n-1}^{t} \Sigma(s) d B(s)-\int_{n-1}^{t} K_{1}(s) d s X_{\infty} .
$$

Taking norms on both sides of (4.34), squaring both sides, taking suprema, before finally taking expectations yields:

$$
\begin{array}{rl}
\mathbb{E}\left[\sup _{n-1 \leq t \leq n}\|\Delta(t)\|^{2}\right] \leq 4 & \mathbb{E}\|\Delta(n-1)\|^{2}+\mathbb{E}\left[\left(\int_{n-1}^{n}\|A\|\|\Delta(s)\|+(\|K\| *\|\Delta\|)(s) d s\right)^{2}\right] \\
& \left.+\mathbb{E}\left[\sup _{n-1 \leq t \leq n}\left\|\int_{n-1}^{t} \Sigma(s) d B(s)\right\|^{2}\right]+\left(\int_{n-1}^{n}\left\|K_{1}(s)\right\| d s\right)^{2} \mathbb{E}\left\|X_{\infty}\right\|^{2}\right\} .
\end{array}
$$

We now consider each term on the right hand side of (4.35). From Lemma 4.3 we see that the first term satisfies

$$
\mathbb{E}\left[\|\Delta(n-1)\|^{2}\right] \leq m\left(X_{0}\right) e^{-2 \lambda(n-1)} .
$$

In order to obtain an exponential bound on the second term on the right hand side of (4.26) we make use of the Cauchy-Schwarz inequality as follows:

$$
\begin{aligned}
& \left(\int_{n-1}^{n}\|A\|\|\Delta(s)\|+(\|K\| *\|\Delta\|)(s) d s\right)^{2} \\
& \quad \leq 2 \int_{n-1}^{n}\left(\|A\|^{2}\|\Delta(s)\|^{2}+(\|K\| *\|\Delta\|)^{2}(s)\right) d s \\
& \quad \leq 2 \int_{n-1}^{n}\left[\|A\|^{2}\|\Delta(s)\|^{2}+\left(\int_{0}^{s} e^{\alpha(s-u) / 2}\|K(s-u)\|^{1 / 2} e^{-\alpha(s-u) / 2}\|K(s-u)\|^{1 / 2}\|\Delta(s)\| d u\right)^{2}\right] d s \\
& \quad \leq 2 \int_{n-1}^{n}\left[\|A\|^{2}\|\Delta(s)\|^{2}+\bar{K}_{\alpha} \int_{0}^{s} e^{-\alpha(s-u)}\|K(s-u)\|\|\Delta(s)\|^{2} d u\right] d s,
\end{aligned}
$$


where $\bar{K}_{\alpha}=\int_{0}^{\infty} e^{\alpha t}\|K(t)\| d t$. Take expectations and examine the two terms within the integral. Using Lemma 4.3 we obtain

$$
\mathbb{E}\left[\int_{n-1}^{n}\|A\|^{2}\|\Delta(s)\|^{2} d s\right] \leq\|A\|^{2} m\left(X_{0}\right) \int_{n-1}^{n} e^{-2 \lambda s} d s \leq c_{1}\left(X_{0}\right) e^{-2 \lambda(n-1)} .
$$

In order to obtain an exponential upper bound for the second term within the integral we apply Lemma 4.11 with $K=\tilde{K}, \alpha=\tilde{\alpha}, \lambda=\tilde{\lambda}$ and $\eta=\tilde{\eta}$ :

$$
\begin{aligned}
\mathbb{E}\left[\int_{n-1}^{n} \bar{K}_{\alpha} \int_{0}^{s} e^{-\alpha(s-u)}\|K(s-u)\|\|\Delta(s)\|^{2} d u d s\right] & \leq m\left(X_{0}\right) \bar{K}_{\alpha} \int_{n-1}^{n} \int_{0}^{s} e^{-\alpha u}\|K(u)\| e^{-2 \lambda(s-u)} d u d s \\
& \leq c_{2}\left(X_{0}\right) e^{-\eta(n-1)} .
\end{aligned}
$$

Next, we obtain an exponential upper bound on the third term. Using (4.21) and the Burkholder-Davis-Gundy inequality, there exists a constant $c_{3}>0$ such that

$$
\mathbb{E}\left[\sup _{n-1 \leq t \leq n}\left\|\int_{n-1}^{t} \Sigma(s) d B(s)\right\|^{2}\right] \leq c_{3} \bar{\Sigma} e^{-2 \gamma(n-1)} .
$$

Now consider the last term on the right hand side of (4.35). Using (3.4) we see that

$$
\bar{K}_{\alpha}:=\int_{0}^{\infty}\|K(s)\| e^{\alpha s} d s \geq e^{\alpha t} \int_{t}^{\infty}\|K(s)\| d s \geq e^{\alpha t}\left\|K_{1}(t)\right\| .
$$

Using this and the fact that $\mathbb{E}\left\|X_{\infty}\right\|^{2}<\infty$ (see (4.16)) we obtain

$$
\left(\int_{n-1}^{n}\left\|K_{1}(s)\right\| d s\right)^{2} \mathbb{E}\left\|X_{\infty}\right\|^{2} \leq \mathbb{E}\left\|X_{\infty}\right\|^{2}\left(\int_{n-1}^{n} \bar{K}_{\alpha} e^{-\alpha s} d s\right)^{2} \leq c_{4} e^{-2 \alpha(n-1)} .
$$

Combining (4.36), (4.38), (4.39), (4.40), and (4.42) we obtain

$$
\mathbb{E}\left[\sup _{n-1 \leq t \leq n}\left\|X(t)-X_{\infty}\right\|^{2}\right] \leq \tilde{m}\left(X_{0}\right) e^{-2 \eta(n-1)},
$$

where $\tilde{m}\left(X_{0}\right)=4\left(m\left(X_{0}\right)+c_{1}\left(X_{0}\right)+c_{2}\left(X_{0}\right)+c_{3} \bar{\Sigma}+c_{4}\right)$ and $\eta<\min (2 \lambda, \alpha)$.

We can now apply the line of reasoning used in [10, Theorem 4.4.2] to obtain (3.10).

Proof of Lemma 4.4. We use a reformulation of (1.2a)-(1.2b) in the proof of this result. It is obtained as follows: multiply both sides of $R^{\prime}(s)=A R(s)+(K * R)(s)$ across by the function $\Phi(t-s)$, where $\Phi(t)=P+e^{-t} Q$, integrate over $[0, t]$, use integration by parts, add and subtract $R_{\infty}$ from both sides to obtain

$$
Y(t)+(F * Y)(t)=G(t), \quad t \geq 0,
$$

where $Y=R-R_{\infty}, F$ is defined by (4.12) and $G$ is defined by

$$
\begin{aligned}
G(t)= & e^{-t} Q-e^{-t}\left(Q R_{\infty}+Q A R_{\infty}\right)+\int_{t}^{\infty} \int_{s}^{\infty} P K(u) R_{\infty} d u d s \\
& -\int_{t}^{\infty} Q K(u) R_{\infty} d u-\left(e * Q K R_{\infty}\right)(t), \quad t \geq 0 .
\end{aligned}
$$


Consider the reformulation of (1.2a)-(1.2b) given by (4.44). It is well known that $Y$ can be expressed as

$$
Y(t)=G(t)-\int_{0}^{t} r(t-s) G(s) d s
$$

where the function $r$ satisfies $r+F * r=F$ and $r+r * F=F$. We refer the reader to [11] for details. Consider the first term on the right hand side of (4.46). As (3.1) holds it is clear that the function $G$ is integrable. Now consider the second term. Since (3.3) and (4.4) hold we may apply Lemma 4.9 to obtain (4.11). Now we may apply a result of Paley and Wiener (see [11]) to see that $r$ is integrable. The convolution of an integrable function with an integrable function is itself integrable. Now combining the arguments for the first and second terms we see that (4.2) must hold.

\section{On the necessity of $(3.5)$ for exponential convergence of solutions of $(1.4 a)-(1.4 b)$}

In this section, the necessity of condition (3.5) for exponential convergence in the almost sure and $p$ th mean senses is shown. Proposition 5.1 concerns the necessity of the condition in the almost sure case while Proposition 5.2 deals with the $p$ th mean case.

Proposition 5.1. Let $K$ satisfy (2.4) and (4.4) and $\Sigma$ satisfy (2.6). If there exists a constant $\alpha>0$ such that (3.4) holds, and if for all $X_{0}$ there is a constant vector $X_{\infty}\left(X_{0}, \Sigma\right)$ such that the solution $t \mapsto X\left(t ; X_{0}, \Sigma\right)$ of (1.4a)-(1.4b) satisfies statement (iii) of Theorem 3.2, then there exists a constant $\gamma>0$, independent of $X_{0}$, such that (3.5) holds.

Proposition 5.2. Let $K$ satisfy (2.4) and (4.4) and $\Sigma$ satisfy (2.6). If there exists a constant $\alpha>0$ such that (3.4) holds, and if for all $X_{0}$ there is a constant vector $X_{\infty}\left(X_{0}, \Sigma\right)$ such that the solution $t \mapsto X\left(t ; X_{0}, \Sigma\right)$ of $(1.4 \mathrm{a})-(1.4 \mathrm{~b})$ satisfies statement (ii) of Theorem 3.2, then there exists a constant $\gamma>0$, independent of $X_{0}$, such that (3.5) holds.

In order to prove these propositions the integral version of (1.4a)-(1.4b) is considered. By reformulating this version of the equation an expression for a term related to the exponential integrability of the perturbation is found. Using various arguments, including the Martingale Convergence Theorem in the almost sure case, this term is used to show that (3.5) holds.

Some supporting results are now stated. Lemma 5.3 is the analogue of Lemma 4.7 in the mean square case. It was proved in [8].

Lemma 5.3. Let $K$ satisfy (2.4) and (4.4). Suppose that for all initial conditions $X_{0}$ there is a $\mathcal{F}^{B}(\infty)$ measurable and almost surely finite random variable $X_{\infty}\left(X_{0}, \Sigma\right)$ with $\mathbb{E}\left\|X_{\infty}\right\|^{2}<\infty$ such that the solution $t \mapsto X\left(t ; X_{0}, \Sigma\right)$ of $(1.4 \mathrm{a})-(1.4 \mathrm{~b})$ satisfies

$$
\begin{gathered}
\lim _{t \rightarrow \infty} \mathbb{E}\left\|X\left(t ; X_{0}, \Sigma\right)-X_{\infty}\left(X_{0}, \Sigma\right)\right\|^{2}=0, \\
\mathbb{E}\left\|X\left(\cdot ; X_{0}, \Sigma\right)-X_{\infty}\left(X_{0}, \Sigma\right)\right\|^{2} \in L^{1}([0, \infty), \mathbb{R}) .
\end{gathered}
$$

Then $\mathrm{X}_{\infty}$ obeys

$$
\left(A+\int_{0}^{\infty} K(s) d s\right) X_{\infty}=0 \text { a.s. }
$$


Lemma 5.4 may be extracted from [4]; it is required in the proof of Proposition 5.2.

Lemma 5.4. Let $N=\left(N_{1}, \ldots, N_{n}\right)$ where $N_{i} \sim \mathcal{N}\left(0, v_{i}^{2}\right)$ for $i=1, \ldots, n$. Then there exists $a\left\{v_{i}\right\}_{i=1^{-}}^{n}$ independent constant $d_{1}>0$ such that

$$
\mathbb{E}\left[\|N\|^{2}\right] \leq d_{1} \mathbb{E}[\|N\|]^{2} .
$$

Proof of Proposition 5.1. In order to prove this result we follow the argument used in [4, Theorem 4.1]. Let $0<\gamma<\alpha \wedge \beta_{0}$. By defining the process $Z(t)=e^{\gamma t} X(t)$ and the matrix $\kappa(t)=e^{\gamma t} K(t)$ we can rewrite $(1.4 \mathrm{a})-(1.4 \mathrm{~b})$ as

$$
d Z(t)=\left((\gamma I+A) Z(t)+\int_{0}^{t} \kappa(t-s) Z(s) d s\right) d t+e^{\gamma t} \Sigma(t) d B(t)
$$

the integral form of which is

$$
Z(t)-Z(0)=(\gamma I+A) \int_{0}^{t} Z(s) d s+\int_{0}^{t} \int_{0}^{s} \kappa(s-u) Z(u) d u d s+\int_{0}^{t} e^{\gamma s} \Sigma(s) d B(s) .
$$

Using $Z(t)=e^{\gamma t} X(t)$ and rearranging this becomes

$$
\int_{0}^{t} e^{r s} \Sigma(s) d B(s)=e^{\gamma t} X(t)-X_{0}-(\gamma I+A) \int_{0}^{t} e^{\gamma s} X(s) d s-\int_{0}^{t} e^{r s} \int_{0}^{s} K(s-u) X(u) d u d s .
$$

Adding and subtracting $X_{\infty}$ from the right hand side and applying Lemma 4.7 we obtain:

$$
\begin{aligned}
\int_{0}^{t} e^{\gamma s} \Sigma(s) d B(s)= & e^{\gamma t}\left(X(t)-X_{\infty}\right)-\left(X_{0}-X_{\infty}\right)-(\gamma I+A) \int_{0}^{t} e^{\gamma s}\left(X(s)-X_{\infty}\right) d s \\
& -\int_{0}^{t} e^{\gamma s} \int_{0}^{s} K(s-u)\left(X(u)-X_{\infty}\right) d u d s+\int_{0}^{t} e^{r s} K_{1}(s) d s X_{\infty} .
\end{aligned}
$$

Consider each term on the right hand side of (5.7). We see that the first term tends to zero as (3.10) holds and $\gamma<\beta_{0}$. The second term is finite by hypothesis. Again, using the fact that $\gamma<\beta_{0}$ and that assumption (3.10) holds we see that $e_{\gamma}\left(X-X_{\infty}\right) \in L^{1}[0, \infty)$, so the third term tends to a limit as $t \rightarrow \infty$. Now consider the fourth term. Since $0<\gamma<\alpha \wedge \beta_{0}$, we can choose $\gamma_{1}>0$ such that $\gamma<\gamma_{1}<\alpha \wedge \beta_{0}$. So the functions $t \mapsto e^{\gamma_{1} t} K(t)$ and $t \mapsto e^{\gamma_{1} t}\left(X(t)-X_{\infty}\right)$ are both integrable. The convolution of these two integrable functions is itself an integrable function, so

$$
\left\|\int_{0}^{s} K(s-u)\left(X(u)-X_{\infty}\right) d u\right\| \leq c e^{-\gamma_{1} s} .
$$

Thus, it is clear that the fourth term has a finite limit as $t \rightarrow \infty$. Finally, the fifth term on the right hand side of (5.7) has a finite limit at infinity, using (4.41). therefore

Each term on the right hand side of the inequality has a finite limit as $t \rightarrow \infty$, so

$$
\lim _{t \rightarrow \infty} \int_{0}^{t} e^{r s} \Sigma(s) d B(s) \text { exists and is almost surely finite. }
$$

The Martingale Convergence Theorem [12, Proposition 5.1.8] may now be applied component by component to obtain (3.5). 
Proof of Proposition 5.2. By Lemma 5.3, (5.7) still holds. Define $\gamma<\alpha \wedge \beta_{1}$, take norms and expectations across (5.7) to obtain

$$
\begin{aligned}
\mathbb{E}\left[\left\|\int_{0}^{t} e^{r s} \Sigma(s) d B(s)\right\|\right] \leq & \mathbb{E}\left[e^{\gamma t}\left\|X(t)-X_{\infty}\right\|\right]+\mathbb{E}\left[\left\|X_{0}-X_{\infty}\right\|\right] \\
& +\|\gamma I+A\| \int_{0}^{t} \mathbb{E}\left[e^{\gamma s}\left\|X(s)-X_{\infty}\right\|\right] d s \\
& +\int_{0}^{t} e^{\gamma s} \int_{0}^{s}\|K(u)\| \mathbb{E}\left[\left\|X(s-u)-X_{\infty}\right\|\right] d u d s \\
& +\int_{0}^{t} e^{\gamma s}\left\|K_{1}(s)\right\| d s \mathbb{E}\left\|X_{\infty}\right\| .
\end{aligned}
$$

There exists $m_{1}$ such that

$$
\mathbb{E}\left[e^{\gamma t}\left\|X(t)-X_{\infty}\right\|\right] \leq m_{1} e^{-\left(\beta_{1}-\gamma\right) t},
$$

thus the first, second and third terms on the right hand side of (5.10) are uniformly bounded on $[0, \infty)$. Now consider the fourth term. Since $0<\gamma<\alpha \wedge \beta_{1}$, we can choose $\gamma_{1}>0$ such that $\gamma<\gamma_{1}<\alpha \wedge \beta_{1}$ so that the functions $t \mapsto e^{\gamma_{1} t} K(t)$ and $t \mapsto e^{\gamma_{1} t} \mathbb{E}\left\|X(t)-X_{\infty}\right\|$ are both integrable. The convolution of two integrable functions is itself an integrable function, so

$$
\int_{0}^{s}\|K(s-u)\| \mathbb{E}\left\|X(u)-X_{\infty}\right\| d u \leq c e^{-\gamma_{1} s},
$$

so it is clear that the fourth term is uniformly bounded on $[0, \infty)$. Finally, we consider the final term on the right hand side of (5.10). Using (4.41) we obtain

$$
\int_{0}^{t} e^{r s}\left\|K_{1}(s)\right\| d s \mathbb{E}\left\|X_{\infty}\right\| \leq \bar{K}_{\alpha} \mathbb{E}\left\|X_{\infty}\right\| \int_{0}^{t} e^{-(\alpha-\gamma) s} d s<\infty,
$$

since $\gamma<\alpha$. Thus there is a constant $c>0$ such that

$$
\mathbb{E}\left[\left\|\int_{0}^{t} e^{r s} \Sigma(s) d B(s)\right\|\right] \leq c .
$$

The proof now follows the line of reasoning found in [4, Theorem 4.3]: observe that

$$
\left\|\int_{0}^{t} e^{r s} \Sigma(s) d B(s)\right\|^{2}=\sum_{i=1}^{n} N_{i}(t)^{2},
$$

where

$$
N_{i}(t)=\sum_{j=1}^{d} \int_{0}^{t} e^{r s} \Sigma_{i j}(s) d B_{j}(s) .
$$


It is clear that $N_{i}(t)$ is normally distributed with zero mean and variance given by

$$
v_{i}(t)^{2}=\sum_{j=1}^{d} \int_{0}^{t} e^{2 \gamma s} \Sigma_{i j}(s)^{2} d s
$$

Lemma 5.4 and (5.14) may now be applied to obtain:

$$
\begin{aligned}
\int_{0}^{t} e^{2 \gamma s}\|\Sigma(s)\|^{2} d s & =\sum_{i=1}^{n} \sum_{j=1}^{d} \int_{0}^{t} e^{2 \gamma s}\left|\Sigma_{i j}(s)\right|^{2} d s \\
& =\sum_{i=1}^{n} v_{i}(t)^{2} \\
& \left.=\mathbb{E}\left[\left\|\int_{0}^{t} e^{r s} \Sigma(s) d B(s)\right\|\right]^{2}\right] \\
& \leq d_{1} \mathbb{E}\left[\left\|\int_{0}^{t} e^{r s} \Sigma(s) d B(s)\right\|\right]^{2} \leq d_{1} c^{2} .
\end{aligned}
$$

Allowing $t \rightarrow \infty$ on both sides of this inequality yields the desired result.

\section{Sufficient conditions for exponential convergence of solutions of $(1.1 \mathrm{a})-(1.1 \mathrm{~b})$}

In this section, sufficient conditions for exponential convergence of solutions of (1.1a)(1.1b) to a nontrivial limit are found. Proposition 6.1 concerns the $p$ th mean sense while Proposition 6.2 deals with the almost sure case.

Proposition 6.1. Let $K$ satisfy (2.4) and (3.1), let $\Sigma$ satisfy (2.6), let $f$ satisfy (2.5), and let $R_{\infty}$ be a constant matrix such that the solution $R$ of (1.2a)-(1.2b) satisfies (3.3). If there exist constants $\alpha>0$, $\rho>0$ and $\gamma>0$ such that (3.4), (3.6) and (3.5) hold, then there exist constants $\beta_{p}^{*}>0$, independent of $X_{0}$, and $m_{p}^{*}=m_{p}^{*}\left(X_{0}\right)>0$, such that statement (ii) of Theorem 3.1 holds.

Proposition 6.2. Let $K$ satisfy (2.4) and (3.1), let $\Sigma$ satisfy (2.6), let $f$ satisfy (2.5), and let $R_{\infty}$ be a constant matrix such that the solution $R$ of (1.2a)-(1.2b) satisfies (3.3). If there exist constants $\alpha>0, \rho>0$ and $\gamma>0$ such that (3.4), (3.6) and (3.5) hold, then there exists constant $\beta_{0}^{*}>0$, independent of $X_{0}$ such that statement (iii) of Theorem 3.1 holds.

As in the case where $f:=0$ we require an explicit formulation for $X_{\infty}$. The proof of this result follows the line of reasoning used in the proof of Theorem 4.6 and is therefore omitted.

Theorem 6.3. Let $K$ satisfy (2.4) and (4.4), let $\Sigma$ satisfy (2.6) and (4.5), and let $f$ satisfy (2.5). Suppose that the resolvent $R$ of $(1.2 \mathrm{a})-(1.2 \mathrm{~b})$ satisfies (3.3), then the solution $X$ of (1.1a)-(1.1b) satisfies $X \rightarrow X_{\infty}\left(X_{0}, \Sigma, f\right)$ almost surely, where

$$
X_{\infty}\left(X_{0}, \Sigma, f\right)=X_{\infty}\left(X_{0}, \Sigma\right)+R_{\infty} \int_{0}^{\infty} f(t) d t, \text { a.s. }
$$

and $X_{\infty}\left(X_{0}, \Sigma, f\right)$ is almost surely finite. 
Proof of Proposition 6.1. We begin by showing that $\mathbb{E}\left\|X_{\infty}\left(X_{0}, \Sigma, f\right)\right\|^{p}$ is finite. Clearly, we see that

$$
\mathbb{E}\left\|X_{\infty}\left(X_{0}, \Sigma, f\right)\right\|^{p} \leq 2^{p-1}\left(\mathbb{E}\left\|X_{\infty}\left(X_{0}, \Sigma\right)\right\|^{p}+\left\|\int_{0}^{\infty} R_{\infty} f(s) d s\right\|^{p}\right)<\infty .
$$

Now, consider the difference between the solution $X\left(\cdot ; X_{0}, \Sigma, f\right)$ of $(1.1 \mathrm{a})-(1.1 \mathrm{~b})$ and its limit $X_{\infty}\left(X_{0}, \Sigma, f\right)$ given by $(6.1)$ :

$$
\begin{aligned}
& X\left(t ; X_{0}, \Sigma, f\right)-X_{\infty}\left(X_{0}, \Sigma, f\right) \\
& \quad=\left(X\left(t ; X_{0}, \Sigma\right)-X_{\infty}\left(X_{0}, \Sigma\right)\right)+\int_{0}^{t}\left(R(t-s)-R_{\infty}\right) f(s) d s-\int_{t}^{\infty} R_{\infty} f(s) d s .
\end{aligned}
$$

Using integration by parts this expression becomes

$$
\begin{aligned}
& X\left(t ; X_{0}, \Sigma, f\right)-X_{\infty}\left(X_{0}, \Sigma, f\right) \\
& \quad=\left(X\left(t ; X_{0}, \Sigma\right)-X_{\infty}\left(X_{0}, \Sigma\right)\right)-f_{1}(t)+\left(R(t)-R_{\infty}\right) f_{1}(0)-\int_{0}^{t} R^{\prime}(t-s) f_{1}(s) d s
\end{aligned}
$$

Taking norms on both sides of equation (6.4), raising the power to $p$ on both sides, and taking expectations across we obtain

$$
\begin{aligned}
& \mathbb{E}\left\|X\left(t ; X_{0}, \Sigma, f\right)-X_{\infty}\left(X_{0}, \Sigma, f\right)\right\|^{p} \\
& \leq 4^{p-1}\left(\mathbb{E}\left\|X\left(t ; X_{0}, \Sigma\right)-X_{\infty}\left(X_{0}, \Sigma\right)\right\|^{p}+\left\|f_{1}(t)\right\|^{p}\right. \\
&\left.\quad+\left\|R(t)-R_{\infty}\right\|^{p}\left\|f_{1}(0)\right\|^{p}+\left(\int_{0}^{t}\left\|R^{\prime}(t-s)\right\|\left\|f_{1}(s)\right\| d s\right)^{p}\right) .
\end{aligned}
$$

Now consider the right hand side of (6.5). The first term decays exponentially quickly due to Theorem 3.2. The second term decays exponentially quickly due to assumption (3.6). By applying Lemma 4.4 we see that (4.2) holds so we can apply Theorem 4.5 to show that the third term must decay exponentially. In the sequel, an argument is provided to show that $R^{\prime}$ decays exponentially; thus the final term must decay exponentially. Combining these arguments we see that (3.7) holds, where $\beta_{p}^{*}<\min \left(\beta_{p}, \beta, \rho\right)$.

It is now shown that $R^{\prime}$ decays exponentially. It is clear from the resolvent equation (1.2a)-(1.2b) that

$$
R^{\prime}(t)=A\left(R(t)-R_{\infty}\right)+\int_{0}^{t} K(t-s)\left(R(s)-R_{\infty}\right) d s-K_{1}(t) R_{\infty}+\left(A+\int_{0}^{\infty} K(s) d s\right) R_{\infty} .
$$

Consider each term on the right hand side of (6.6). We can apply Theorem 4.5 to obtain that $R$ decays exponentially quickly to $R_{\infty}$. In order to show that the second term decays exponentially we proceed as follows: since $R-R_{\infty}$ decays exponentially and (3.4) holds it is possible to choose $\mu$ such that the functions $t \mapsto e^{\mu t} K(t)$ and $t \mapsto e^{\mu t}\left(R(t)-R_{\infty}\right)$ are both in $L^{1}\left([0, \infty), \mathbb{R}^{n \times n}\right)$. The convolution of two integrable functions is itself an integrable function, so

$$
e^{\mu t}\left\|\int_{0}^{t} K(t-s)\left(R(s)-R_{\infty}\right) d s\right\|=\left\|\int_{0}^{t} e^{\mu(t-s)} K(t-s) e^{\mu s}\left(R(s)-R_{\infty}\right) d s\right\| \leq c .
$$


To see that the third term decays exponentially we use (4.41). Finally, we consider the fourth term. By Lemma 4.4 and (3.3) we have that (4.2) holds. In [1, Theorem 6.1] it was shown that $\left(A+\int_{0}^{\infty} K(s) d s\right) R_{\infty}=0$ under this hypothesis and (3.1). Combining the above we see that $R^{\prime}$ decays exponentially quickly to 0 .

Proof of Proposition 6.2. Take norms across (6.4) to obtain

$$
\begin{aligned}
& \left\|X\left(t ; X_{0}, \Sigma, f\right)-X_{\infty}\left(X_{0}, \Sigma, f\right)\right\| \\
& \quad \leq\left\|X\left(t ; X_{0}, \Sigma\right)-X_{\infty}\left(X_{0}, \Sigma\right)\right\|+\left\|f_{1}(t)\right\|+\left\|R(t)-R_{\infty}\right\|\left\|f_{1}(0)\right\|+\left\|\int_{0}^{t} R^{\prime}(t-s) f_{1}(s) d s\right\| .
\end{aligned}
$$

Using Theorem 3.2, we see that the first term on the right hand side of (6.8) decays exponentially. The second term on the right hand side decays exponentially as (3.6) holds. We can apply Theorem 4.5 to show that the third term must decay exponentially. An argument was provided in Proposition 6.1 to show that $R^{\prime}$ decays exponentially. Combining this with (3.6) enables us to show that the fourth term decays exponentially. Using the above arguments we obtain (3.8), where $\beta^{*} \leq \min \left(\beta_{0}, \beta, \rho\right)$.

\section{On the necessity of (3.6) and (3.5) for exponential convergence of solutions of $(1.1 \mathrm{a})-(1.1 \mathrm{~b})$}

In this section, the necessity of (3.6) and (3.5) for exponential convergence of solutions of (1.1a)-(1.1b) in the almost sure and $p$ th mean senses is shown. Proposition 7.1 concerns the necessity of the conditions in the $p$ th mean case while Proposition 7.2 deals with the almost sure case.

Proposition 7.1. Let $K$ satisfy (2.4) and (4.4), let $\Sigma$ satisfy (2.6), and let $f$ satisfy (2.5). If there exists constant $\alpha>0$ such that (3.4) holds, and if for all $X_{0}$ there is constant vector $X_{\infty}\left(X_{0}, \Sigma, f\right)$ such that the solution $t \mapsto X\left(t ; X_{0}, \Sigma, f\right)$ of (1.1a)-(1.1b) satisfies statement (ii) of Theorem 3.1, then there exist constants $\rho>0$ and $\gamma>0$, independent of $X_{0}$, such that (3.6) and (3.5) hold.

Proposition 7.2. Let $K$ satisfy (2.4) and (4.4), let $\Sigma$ satisfy (2.6), and let $f$ satisfy (2.5). If there exists constant $\alpha>0$ such that (3.4) holds, and if for all $X_{0}$ there is a constant vector $X_{\infty}\left(X_{0}, \Sigma, f\right)$ such that the solution $t \mapsto X\left(t ; X_{0}, \Sigma, f\right)$ of (1.1a)-(1.1b) satisfies statement (iii) of Theorem 3.1, then there exist constants $\rho>0$ and $\gamma>0$, independent of $X_{0}$, such that (3.6) and (3.5) hold.

The following lemma is used in the proof of Proposition 7.2. This lemma allows us to separate the behavior of the deterministic perturbation from the stochastic perturbation in the almost sure case. It is interesting to note that we can prove this lemma without any reference to the integro-differential equation.

Lemma 7.3. Suppose $c>0$ is an almost surely finite random variable and

$$
\left\|f_{1}(t)+\mu_{1}(t, \omega)\right\| \leq c(\omega) e^{-\lambda t},
$$

where $\lambda>0, \omega \in \Omega^{*}, \mathbb{P}\left[\Omega^{*}\right]=1$ and the functions $f_{1}$ and $\mu_{1}$ are defined by (2.8) and

$$
\mu_{1}(t)=\int_{t}^{\infty} \Sigma(s) d B(s), \quad t \geq 0,
$$

respectively. Then (3.5) and (3.6) hold. 
In order to prove Lemma 7.3 we require Lemmas 7.4 and 7.5 below. Lemma 7.5 was proved in [13]. The proof of Lemma 7.4 makes use of Kolmogorov's Zero-One Law. It follows the proof of Theorem 2 in [14, Chapter IV, Section 1] and so is omitted.

Lemma 7.4. Let $\left\{\xi_{i}\right\}_{i=1}^{\infty}$ be a sequence of independent Gaussian random variables with $\mathbb{E}\left[\xi_{i}\right]=0$ and $\mathbb{E}\left[\xi_{i}^{2}\right]=v_{i}^{2} \geq 1$. Then

$$
\limsup _{m \rightarrow \infty} \sum_{i=1}^{m} \xi_{i}=\infty, \quad \liminf _{m \rightarrow \infty} \sum_{i=1}^{m} \xi_{i}=-\infty \text {, a.s. }
$$

Lemma 7.5. If there is a $\gamma>0$ such that $\sigma \in C([0, \infty), \mathbb{R})$ and

$$
\int_{0}^{\infty} \sigma(s)^{2} e^{2 \gamma s} d s<\infty
$$

then

$$
\limsup _{t \rightarrow \infty} \frac{1}{t} \log \left|\int_{t}^{\infty} \sigma(s) d B(s)\right| \leq-\gamma, \text { a.s. }
$$

where $\{B(t)\}_{t \geq 0}$ is a one-dimensional standard Brownian motion.

Lemmas 7.6 and 7.7 are used in the proofs of Propositions 7.1 and 7.2, respectively and are the analogues of Lemmas 5.3 and 4.7. Their proofs are identical in all important aspects and so are omitted.

Lemma 7.6. Let $K$ satisfy (2.4) and (4.4). Suppose that for all initial conditions $X_{0}$ there is an $\Psi^{B}(\infty)$-measurable and almost surely finite random variable $X_{\infty}\left(X_{0}, \Sigma\right)$ with $\mathbb{E}\left\|X_{\infty}\right\|^{2}<\infty$ such that the solution $t \mapsto X\left(t ; X_{0}, \Sigma\right)$ of $(1.1 \mathrm{a})-(1.1 \mathrm{~b})$ satisfies

$$
\begin{gathered}
\lim _{t \rightarrow \infty} \mathbb{E}\left\|X\left(t ; X_{0}, \Sigma, f\right)-X_{\infty}\left(X_{0}, \Sigma, f\right)\right\|^{2}=0, \\
\mathbb{E}\left\|X\left(\cdot ; X_{0}, \Sigma, f\right)-X_{\infty}\left(X_{0}, \Sigma, f\right)\right\|^{2} \in L^{1}([0, \infty), \mathbb{R}) .
\end{gathered}
$$

Then $\mathrm{X}_{\infty}$ obeys

$$
\left(A+\int_{0}^{\infty} K(s) d s\right) X_{\infty}=0 \text { a.s. }
$$

Lemma 7.7. Let $K$ satisfy (2.4) and (4.4). Suppose that for all initial conditions $X_{0}$ there is an $F^{B}(\infty)$-measurable and almost surely finite random variable $X_{\infty}\left(X_{0}, \Sigma, f\right)$ such that the solution $t \mapsto X\left(t ; X_{0}, \Sigma, f\right)$ of (1.1a)-(1.1b) satisfies

$$
\begin{gathered}
\lim _{t \rightarrow \infty} X\left(t ; X_{0}, \Sigma, f\right)=X_{\infty}\left(X_{0}, \Sigma, f\right) \text { a.s., } \\
X\left(\cdot ; X_{0}, \Sigma, f\right)-X_{\infty}\left(X_{0}, \Sigma, f\right) \in L^{2}\left([0, \infty), \mathbb{R}^{n}\right) \text { a.s. }
\end{gathered}
$$

Then $X_{\infty}$ obeys (7.7). 
Proof of Proposition 7.1. Since (3.7) holds for every initial condition we can choose $X_{0}=0$ : this simplifies calculations. Moreover using (3.7) in Lemma 7.6 it is clear that assumption (7.7) holds. Consider the integral form of (1.1a)-(1.1b). Adding and subtracting $X_{\infty}$ from both sides and applying Lemma 7.6 we obtain

$$
\Delta(t)=-X_{\infty}+\int_{0}^{t} \delta(s) d s+\int_{0}^{t} f(s) d s+\mu(t)-\int_{0}^{t} K_{1}(s) d s X_{\infty}
$$

where $\Delta(t)=X(t)-X_{\infty}$, the function $\delta$ is defined by

$$
\delta(t)=A \Delta(t)+(K * \Delta)(t),
$$

and $\mu(t)=\int_{0}^{t} \Sigma(s) d B(s)$. Taking expectations across (7.9) and allowing $t \rightarrow \infty$ we obtain

$$
-\mathbb{E}\left[X_{\infty}\right]=-\int_{0}^{\infty} \mathbb{E}[\delta(s)] d s-\int_{0}^{\infty} f(s) d s+\int_{0}^{\infty} K_{1}(s) d s \mathbb{E}\left[X_{\infty}\right],
$$

where $\mathbb{E}[\delta(t)]=A \mathbb{E}[\Delta(t)]+(K * \mathbb{E}[\Delta])(t)$. Using this expression for $\mathbb{E}\left[X_{\infty}\right]$ we obtain

$$
f_{1}(t)=-\mathbb{E}[\Delta(t)]-\int_{t}^{\infty} \mathbb{E}[\delta(s)] d s+\int_{t}^{\infty} K_{1}(s) d s \mathbb{E}\left[X_{\infty}\right],
$$

The first term on the right-hand side of (7.12) decays exponentially due to (3.7). Assumptions (3.4) and (3.7) imply that $\mathbb{E}[\delta(\cdot)]$ decays exponentially so the second term decays exponentially. The third term on the right-hand side of (7.12) decays exponentially due to the argument given by (4.41). Hence, $f_{1}$ decays exponentially.

Proving that (3.5) holds breaks into two steps. We begin by showing that

$$
\left\|\int_{0}^{\infty} e^{\gamma t} f(t) d t\right\|<\infty,
$$

where $\gamma>0$. By choosing $\gamma<\alpha \wedge \beta_{1}$ we can obtain the following reformulation of (1.1a)-(1.1b) using methods applied in [15, Proposition 5.1]

$$
\begin{aligned}
e^{r t} \Delta(t)= & \Delta(0)+(\gamma I+A) \int_{0}^{t} e^{r s} \Delta(s) d s \\
& +\int_{0}^{t} e^{r s} \int_{0}^{s} K(s-u) \Delta(u) d u d s-\int_{0}^{t} e^{r s} K_{1}(s) d u d s X_{\infty} \\
& +\int_{0}^{t} e^{r s} f(s) d s+\int_{0}^{t} e^{r s} \Sigma(s) d B(s) .
\end{aligned}
$$

Rearranging (7.14), taking expectations and then norms on both sides we can obtain

$$
\begin{aligned}
\left\|\int_{0}^{t} e^{r s} f(s) d s\right\| \leq & \mathbb{E}\left\|e^{\gamma t} \Delta(t)\right\|+\mathbb{E}\|\Delta(0)\| \\
& +\|\gamma I+A\| \int_{0}^{t} e^{r s} \mathbb{E}\|\Delta(s)\| d s \\
& +\int_{0}^{t} e^{r s} \int_{0}^{s}\|K(s-u)\| \mathbb{E}\|\Delta(u)\| d u d s+\int_{0}^{t} e^{r s}\left\|K_{1}(s)\right\| d s \mathbb{E}\left\|X_{\infty}\right\| .
\end{aligned}
$$


Since (3.7) holds this implies that both the first and third terms on the right-hand side of (7.15) are bounded. The second term is bounded due to our assumptions. Since $0<\gamma<\alpha \wedge \beta_{1}$, we can choose $\gamma_{1}>0$ such that $\gamma<\gamma_{1}<\alpha \wedge \beta_{1}$. It can easily be shown that

$$
\int_{0}^{s}\|K(s-u)\| \mathbb{E}[\|\Delta(u)\|] d u \leq c e^{-\gamma_{1} s} .
$$

Finally, we see that the fifth term is bounded using (4.41). So, (7.13) holds.

We now return to (7.14). Again rearranging the equation and taking norms and then expectations across both sides, we obtain

$$
\begin{aligned}
\mathbb{E}\left\|\int_{0}^{t} e^{r s} \Sigma(s) d B(s)\right\| \leq & \mathbb{E}\left\|e^{\gamma t} \Delta(t)\right\|+\mathbb{E}\|\Delta(0)\| \\
& +\|\gamma I+A\| \int_{0}^{t} e^{r s} \mathbb{E}\|\Delta(s)\| d s+\int_{0}^{t} e^{r s} \int_{0}^{s}\|K(s-u)\| \mathbb{E}\|\Delta(u)\| d u d s \\
& +\int_{0}^{t} e^{\gamma s}\left\|K_{1}(u)\right\| d u d s \mathbb{E}\left\|X_{\infty}\right\|+\left\|\int_{0}^{t} e^{r s} f(s) d s\right\| .
\end{aligned}
$$

We already provided an argument above to show that the first five terms on the right hand side of this expression are bounded. Also, we know that (7.13) holds. Thus,

$$
\mathbb{E}\left\|\int_{0}^{t} e^{r s} \Sigma(s) d B(s)\right\| \leq C .
$$

The proof is now identical to Proposition 5.2.

Proof of Proposition 7.2. Since Lemma 7.7 holds we can obtain (7.9). Thus, as $t \rightarrow \infty$, we obtain

$$
-X_{\infty}=-\int_{0}^{\infty} \delta(s) d s-\int_{0}^{\infty} f(s) d s-\mu(\infty)+\int_{0}^{\infty} K_{1}(s) d s X_{\infty}
$$

where $\delta$ is defined by (7.10). Using this expression for $X_{\infty},(7.9)$ becomes

$$
\Delta(t)=-\int_{t}^{\infty} \delta(s) d s-f_{1}(t)-\mu_{1}(t)+\int_{t}^{\infty} K_{1}(s) d s X_{\infty},
$$

where $\mu_{1}(t)=\int_{t}^{\infty} \Sigma(s) d B(s)$. Rearranging the equation and taking norms yields

$$
\left\|f_{1}(t)+\mu_{1}(t)\right\| \leq\|\Delta(t)\|+\left\|\int_{t}^{\infty} \delta(s) d s\right\|+\left\|\int_{t}^{\infty} K_{1}(s) d s X_{\infty}\right\| .
$$

The first term on the right hand side of (7.21) decays exponentially due to (3.8). Using the argument given in (4.41) we see that the third term on the right hand side of (7.21) decays exponentially. Finally, we consider the second term. Clearly $\int_{t}^{\infty} A \Delta(s) d s$ decays exponentially due to (3.8). In order to show that $\int_{t}^{\infty}(K * \Delta)(s) d s$ decays exponentially we use an argument similar to that applied in the proof of Proposition 7.1. So there is an almost surely finite random variable $c>0$ such that

$$
\left\|f_{1}(t)+\mu_{1}(t)\right\| \leq c e^{-\lambda t} \quad \forall t \geq 0, \text { a.s., }
$$

where $\lambda<\min \left(\beta_{0}^{*}, \alpha\right)$. We can now apply Lemma 7.3 to obtain (3.6) and (3.5). 
Proof of Lemma 7.3. We suppose that there exists a constant $\gamma$ such that (3.5) holds. Using the equivalence of norms we see that for all $1 \leq i \leq n$ and $1 \leq j \leq d$ assumption (3.5) implies that

$$
\int_{0}^{\infty} \Sigma_{i j}(s)^{2} e^{2 \gamma s} d s<\infty
$$

Applying Lemma 7.5 we obtain

$$
\limsup _{t \rightarrow \infty} \frac{1}{t} \log \left|\int_{t}^{\infty} \Sigma_{i j}(s) d B_{j}(s)\right| \leq-\gamma, \quad \omega \in \Omega_{i j}, \mathbb{P}\left[\Omega_{i j}\right]=1
$$

Choose any $\epsilon \in(0, \gamma)$. For each $\omega \in \Omega_{i j}$ we can choose a constant $c_{i j}(\omega, \epsilon) \geq 1$ such that

$$
\left|\int_{t}^{\infty} \Sigma_{i j}(s) d B_{j}(s)\right| \leq c_{i j}(\omega, \epsilon) e^{-(\gamma-\epsilon) t}
$$

Now, summing over $j$ we see that

$$
\left|\mu_{1}^{i}(t)\right| \leq c_{i}(\omega, \epsilon) e^{-(\gamma-\epsilon) t},
$$

where $\omega \in \Omega_{i}=\cap_{j=1}^{d} \Omega_{i j}, c_{i}=\sum_{j=1}^{d} c_{i j}$ and $\mu_{1}^{i}(t)=\sum_{j=1}^{d} \int_{t}^{\infty} \Sigma_{i j}(s) d B_{j}(s)$.

Now, since

$$
\left|f_{1}^{i}(t)+\mu_{1}^{i}(t)\right|^{2} \leq \sum_{i=1}^{n}\left|f_{1}^{i}(t)+\mu_{1}^{i}(t)\right|^{2}=\left\|f_{1}(t)+\mu_{1}(t)\right\|^{2}
$$

we see that

$$
\left|f_{1}^{i}(t)+\mu_{1}^{i}(t)\right| \leq c(\omega) e^{-\lambda t}, \quad \omega \in \Omega^{*} .
$$

So for $\omega \in \Omega_{i} \cap \Omega^{*}$ we see that $\left|f_{1}^{i}(t)\right| \leq\left|f_{1}^{i}(t)+\mu_{1}^{i}(t)\right|+\left|\mu_{1}^{i}(t)\right| \leq c(\omega) e^{-\lambda t}+c_{i}(\omega, \epsilon) e^{-(\gamma-\epsilon) t}$. This gives

$$
\left|f_{1}^{i}(t)\right| \leq \bar{c}_{i}(\omega) e^{-\rho t}, \quad \omega \in \Omega^{*} \cap \Omega_{i}
$$

where $\bar{c}_{i}>0$ is finite and $\rho \leq \max (\lambda, \gamma-\epsilon)$. Now summing over $i$ we obtain (3.6), by picking out any $\omega \in \cap_{i}\left(\Omega^{*} \cap \Omega_{i}\right)$. This concludes the case when (3.5) holds.

Now, consider the case where assumption (3.5) fails to hold. We choose a constant $\gamma$ such that $0<\gamma<\ell$ and define the function $d$ as

$$
d(t)=\int_{0}^{t} \frac{1}{r}\left(e^{r s}-1\right) f(s) d s,
$$

and the vector martingale $M$ as

$$
M(t)=\int_{0}^{t} \frac{1}{r}\left(e^{r s}-1\right) \Sigma(s) d B(s) .
$$


We let $M_{i}$ denote the $i$ th component of $M$ and $\left\langle M_{i}\right\rangle$ denote the quadratic variation of $M_{i}$ given by

$$
\left\langle M_{i}\right\rangle(t)=\sum_{j=1}^{d} \int_{0}^{t} \frac{1}{r^{2}}\left(e^{r s}-1\right)^{2} \Sigma_{i j}(s)^{2} d s .
$$

We show at the end of this proof that

$$
\|d(t)+M(t)\|<c^{*}(\omega), \quad \omega \in \Omega^{*}, \quad \mathbb{P}\left[\Omega^{*}\right]=1,
$$

and therefore assume it for the time being.

Since (3.5) fails to hold there exists an entry $i, 1 \leq i \leq n$, of the martingale $M$ such that

$$
\lim _{t \rightarrow \infty}\left\langle M_{i}\right\rangle(t)=\infty
$$

It follows that $\liminf _{t \rightarrow \infty} M_{i}(t)=-\infty$ and $\lim \sup _{t \rightarrow \infty} M_{i}(t)=\infty$ a.s. Consider the corresponding $i$ th entry of $d$, denoted $d_{i}$; it is either bounded or unbounded. If $d_{i}$ is bounded then $M_{i}$ is bounded and so, by the Martingale Convergence Theorem, $\left\langle M_{i}\right\rangle(t)$ is bounded: this contradicts (7.34). So, we suppose the latter, that $d_{i}$ is unbounded, and proceed to show this is also contradictory. Since $\left|d_{i}(t)+M_{i}(t)\right|<c^{*}(\omega)$, for $\omega \in \Omega^{*}$ it is clear that $-c^{*}-M_{i}(t)<d_{i}(t)$. Taking the limit superior on both sides of the inequality yields

$$
\infty=-c^{*}-\liminf _{t \rightarrow \infty} M_{i}(t) \leq \limsup _{t \rightarrow \infty} d_{i}(t) .
$$

As $d$ is deterministic, there exists an increasing sequence of deterministic times $\left\{t_{m}\right\}_{m=0}^{\infty}$ with $t_{0}=0$ such that $d_{i}\left(t_{m}\right) \rightarrow \infty$ as $m \rightarrow \infty$. Consequently, $M_{i}\left(t_{m}\right) \rightarrow-\infty$ as $m \rightarrow \infty$. We choose a subsequence of these times $\left\{\tau_{m}\right\}_{m=0}^{\infty}$ with $\tau_{0}=t_{0}$ such that

$$
v_{l}^{2}:=\sum_{j=1}^{d} \int_{\tau_{l-1}}^{\tau_{l}} \frac{1}{r^{2}}\left(e^{r s}-1\right)^{2} \Sigma_{i j}^{2}(s) d s \geq 1 .
$$

Define $S_{i}(m)=M_{i}\left(\tau_{m}\right)$. Obviously

$$
S_{i}(m)=\sum_{l=1}^{m} \xi_{l}^{(i)}
$$

where

$$
\xi_{l}^{(i)}=\sum_{j=1}^{d} \int_{\tau_{l-1}}^{\tau_{l}} \frac{1}{\gamma}\left(e^{\gamma s}-1\right) \Sigma_{i j}(s) d B_{j}(s) .
$$

It is clear that $\left\{\xi_{l}^{(i)}\right\}_{l=1}^{\infty}$ is an indepenendent normally distributed sequence with the variance of each $\xi_{l}^{(i)}$ given by $v_{l}^{2} \geq 1$ so we may apply Lemma 7.4.

We now show that assumption (7.33) holds. By changing the order of integration we can show that

$$
d(t)=\int_{0}^{t} e^{r s}\left(f_{1}(s)-f_{1}(t)\right) d s, \quad M(t)=\int_{0}^{t} e^{r s}\left(\mu_{1}(s)-\mu_{1}(t)\right) d s .
$$

Thus, as $0<\gamma<\lambda$,

$$
\begin{aligned}
\|d(t)+M(t)\| & \leq \int_{0}^{t} e^{\gamma s}\left(\left\|f_{1}(t)+\mu_{1}(t)\right\|+\left\|f_{1}(s)+\mu_{1}(s)\right\|\right) d s \\
& \leq c(\omega) \int_{0}^{t} e^{\gamma s}\left(e^{-\lambda t}+e^{-\lambda s}\right) d s<c_{1}(\omega), \quad \omega \in \Omega^{*} .
\end{aligned}
$$




\section{On the necessary and sufficient conditions for exponential convergence of solutions of $(1.1 \mathrm{a})-(1.1 \mathrm{~b})$ and $(1.4 \mathrm{a})-(1.4 \mathrm{~b})$}

We now combine the results from Sections 4 and 5 to prove Theorem 3.2 and combine the results from Sections 6 and 7 to prove Theorem 3.1.

We showed the necessity of (3.5) for the exponential convergence of the solution of (1.4a)-(1.4b) in Section 5. In order to prove the necessity of the exponential integrability of the kernel we require the following result which was extracted from [1].

Theorem 8.1. Let $K$ satisfy (2.4) and (3.1). Suppose that there exists a constant matrix $R_{\infty}$ and constants $\beta>0$ and $c>0$ such that the solution $R$ of (1.2a)-(1.2b) satisfies (4.3). If the kernel $K$ satisfies (3.2) then there exists a constant $\alpha>0$ such that $K$ satisfies (3.4).

Proof of Theorem 3.2. We begin by proving the equivalence between (i) and (ii). The implication (i) implies (ii) is the subject of Proposition 4.1. We can demonstrate that (ii) implies (i) as follows. We begin by proving that (3.9) implies (3.4). We consider the following $n$ solutions of $(1.4 \mathrm{a})-(1.4 \mathrm{~b}) ;\left\{X_{j}(t)\right\}_{j=1, \ldots, n^{\prime}}$ where $X_{j}(0)=\mathbf{e}_{j}$. Since (3.9) holds we obtain

$$
m_{1}\left(\mathbf{e}_{j}\right) e^{-\beta_{1} t} \geq \mathbb{E}\left\|X_{j}(t)-X_{j}(\infty)\right\| \geq\left\|\mathbb{E}\left[X_{j}(t)-X_{j}(\infty)\right]\right\|=\left\|(R(t)-R(\infty)) \mathbf{e}_{j}\right\| .
$$

for each $j=1, \ldots, n$. Thus, the resolvent $R$ of (1.2a)-(1.2b) decays exponentially to $R_{\infty}$. We can apply Theorem 8.1 to obtain (3.4) after which Proposition 5.2 can be applied to obtain (3.5). As (8.1) holds it is clear that (3.3) holds.

We now prove the equivalence between (i) and (iii). The implication (i) implies (iii) is the subject of Proposition 4.2. We now demonstrate that (iii) implies (i). We begin by proving that (3.10) implies (3.4). As (3.10) holds for all $X_{0}$ we can consider the following $n+1$ solutions of (1.4a)-(1.4b); $X_{j}(t)_{j=1, \ldots, n+1}$ where

$$
X_{j}(0)=\mathbf{e}_{j} \quad \text { for } j=1, \ldots, n, \quad X_{n+1}(0)=0 .
$$

We know that $X_{j}(t)$ approaches $X_{j}(\infty)$ exponentially quickly in the almost sure sense. Introduce

$$
S_{j}(t)=X_{j}(t)-X_{n+1}(t),
$$

and notice $S_{j}(0)=\mathbf{e}_{j}$. Let $S=\left[S_{1}, \ldots, S_{n}\right] \in \mathbb{R}^{n \times n}$. Then

$$
\begin{gathered}
S^{\prime}(t)=A S(t)+(K * S)(t), \quad t>0, \\
S(0)=I .
\end{gathered}
$$

If we define $S_{j}(\infty)=X_{j}(\infty)-X_{n+1}(\infty)$ then $S(t) \rightarrow S_{\infty}$ exponentially quickly so we can apply Theorem 8.1 to obtain (3.4). As (3.4) and (3.10) hold we can apply Proposition 5.1 to obtain (3.5). Also evident from this argument is that (3.3) holds. This proves that (iii) implies (i).

Proof of Theorem 3.1. We begin by proving the equivalence between (i) and (ii). The implication that (i) implies (ii) is the subject of Proposition 6.1. Now consider the implication (ii) implies (i). Using (3.7) we see that

$$
\left\|\mathbb{E}\left[X(t)-X_{\infty}\right]\right\| \leq \mathbb{E}\left\|X(t)-X_{\infty}\right\| \leq m^{*} e^{-\beta_{1}^{*} t} .
$$


Consider the $n+1$ solutions $X_{j}$ of (1.1a)-(1.1b) with initial conditions $X_{j}(0)=\mathbf{e}_{j}$ for $j=1, \ldots, n$ and $X_{n+1}(0)=0$. Since $R(t) \mathbf{e}_{j}=X_{j}(t)-X_{n+1}(t)$ we see that

$$
\mathbb{E}\left[X_{j}(t)-X_{j}(\infty)\right]+\mathbb{E}\left[X_{n+1}(t)-X_{n+1}(\infty)\right]=R(t) \mathbf{e}_{j}-\mathbb{E}\left[c_{j}\right],
$$

where $c_{j}=X_{j}(\infty)-X_{n+1}(\infty)$ is an almost surely finite constant. As both terms on the left hand side of this expression are decaying exponentially to zero, $t \mapsto R(t) \mathbf{e}_{j}$ must decay exponentially to $\mathbb{E}\left[c_{j}\right]$ as $t \rightarrow \infty$. Thus $R$ must satisfy (4.3). Now, apply Theorem 8.1 to obtain (3.4) and Proposition 7.1 to obtain (3.6) and (3.5).

We now prove the equivalence between (i) and (iii). The implication (i) implies (iii) is the subject of Proposition 6.2. Once again, consider the $n+1$ solutions $X_{j}(t)$ with initial conditions $X_{j}(0)=\mathbf{e}_{j}$ for $j=1, \ldots, n$ and $X_{n+1}(0)=0$. Since $R(t) \mathbf{e}_{j}=X_{j}(t)-X_{n+1}(t)$ for $j=1, \ldots, n$, we can write

$$
\left(X_{j}(t)-X_{j}(\infty)\right)-\left(X_{n+1}(t)-X_{n+1}(\infty)\right)=R(t) \mathbf{e}_{j}-c_{j},
$$

where $c_{j}=X_{j}(\infty)-X_{n+1}(\infty)$ is an almost surely finite random variable. From (3.8) we know that $X_{j}$ decays exponentially quickly to $X_{j}(\infty)$, similarly $X_{n+1}$ decays exponentially quickly to $X_{n+1}(\infty)$. Thus, $R$ decays exponentially to a limit. As a result (4.3) must hold. Now apply Theorem 8.1 to obtain (3.4) and Proposition 7.2 to obtain (3.6) and (3.5).

\section{Acknowledgments}

The authors are pleased to acknowledge the referees for their careful scrutiny of and suggested corrections to the manuscript. The first author was partially funded by an Albert College Fellowship, awarded by Dublin City University's Research Advisory Panel. The second author was funded by The Embark Initiative operated by the Irish Research Council for Science, Engineering and Technology (IRCSET).

\section{References}

[1] J. A. D. Appleby, S. Devin, and D. W. Reynolds, “On the exponential convergence to a limit of solutions of perturbed linear Volterra equations," Electronic Journal of Qualitative Theory of Differential Equations, no. 9, 16 pages, 2005.

[2] S. Murakami, "Exponential asymptotic stability for scalar linear Volterra equations," Differential and Integral Equations, vol. 4, no. 3, pp. 519-525, 1991.

[3] T. Krisztin and J. Terjéki, "On the rate of convergence of solutions of linear Volterra equations," Bollettino della Unione Matemàtica Italiana. Serie VII. B, vol. 2, no. 2, pp. 427-444, 1988.

[4] J. A. D. Appleby and A. Freeman, "Exponential asymptotic stability of linear Itô-Volterra equations with damped stochastic perturbations," Electronic Journal of Probability, vol. 8, no. 22, pp. 223-234, 2003.

[5] J. A. D. Appleby and M. Riedle, "Almost sure asymptotic stability of stochastic Volterra integrodifferential equations with fading perturbations," Stochastic Analysis and Applications, vol. 24, no. 4, pp. 813-826, 2006.

[6] X. Mao, "Stability of stochastic integro-differential equations," Stochastic Analysis and Applications, vol. 18, no. 6, pp. 1005-1017, 2000.

[7] X. Mao and M. Riedle, "Mean square stability of stochastic Volterra integro-differential equations," Systems Control Letters, vol. 55, no. 6, pp. 459-465, 2006.

[8] J. A. D. Appleby, S. Devin, and D. W. Reynolds, "Mean square convergence of solutions of linear stochastic Volterra equations to non-equilibrium limits," Dynamics of Continuous, Discrete Impulsive Systems. Series A, vol. 13B, supplement, pp. 515-534, 2006. 
[9] J. A. D. Appleby, S. Devin, and D. W. Reynolds, "Almost sure convergence of solutions of linear stochastic Volterra equations to nonequilibrium limits," Journal of Integral Equations and Applications, vol. 19, no. 4, pp. 405-437, 2007.

[10] X. Mao, Stochastic Differential Equations and Their Applications, Horwood Publishing Series in Mathematics Applications, Horwood, Chichester, UK, 1997.

[11] G. Gripenberg, S.-O. Londen, and O. Staffans, Volterra Integral and Functional Equations, vol. 34 of Encyclopedia of Mathematics and Its Applications, Cambridge University Press, Cambridge, UK, 1990.

[12] D. Revuz and M. Yor, Continuous Martingales and Brownian Motion, vol. 293 of Fundamental Principles of Mathematical Sciences, Springer, Berlin, Germany, 3rd edition, 1999.

[13] J. A. D. Appleby, "Exponential asymptotic stability of nonlinear Itô-Volterra equations with damped stochastic perturbations," Functional Differential Equations, vol. 12, no. 1-2, pp. 7-34, 2005.

[14] A. N. Shiryaev, Probability, vol. 95 of Graduate Texts in Mathematics, Springer, New York, NY, USA, 2nd edition, 1996

[15] J. A. D. Appleby, S. Devin, and D. W. Reynolds, “On the exponential convergence to a non-equilibruim limit of solutions of linear stochastic Volterra equations," preprint, 2007. 


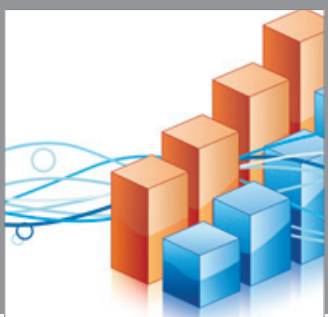

Advances in

Operations Research

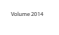

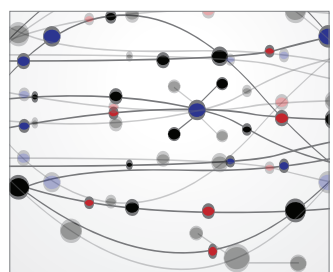

\section{The Scientific} World Journal
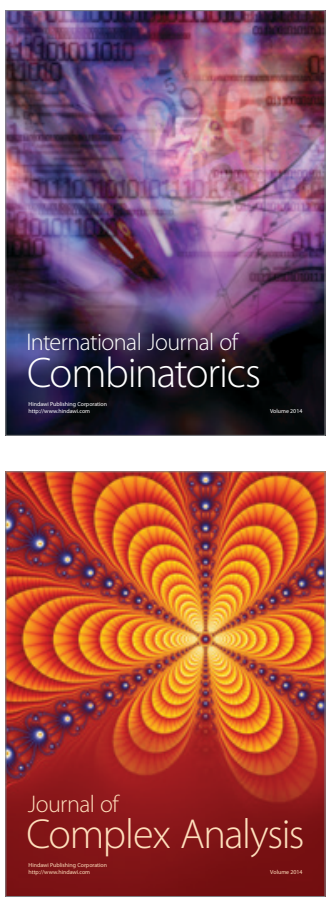

International Journal of

Mathematics and

Mathematical

Sciences
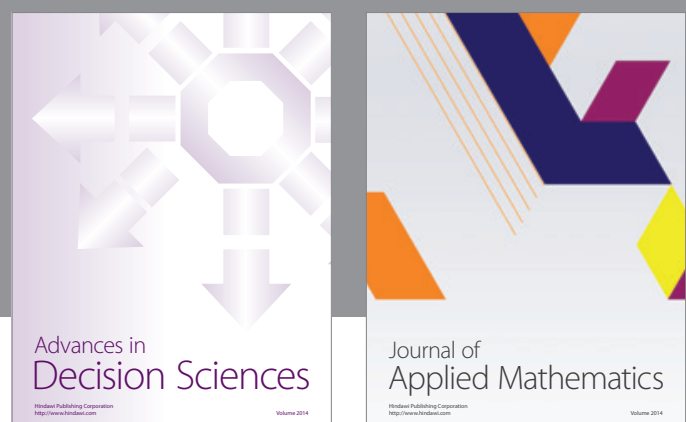

Journal of

Applied Mathematics
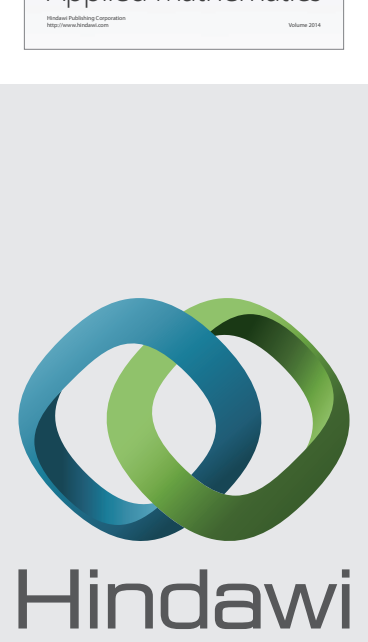

Submit your manuscripts at http://www.hindawi.com
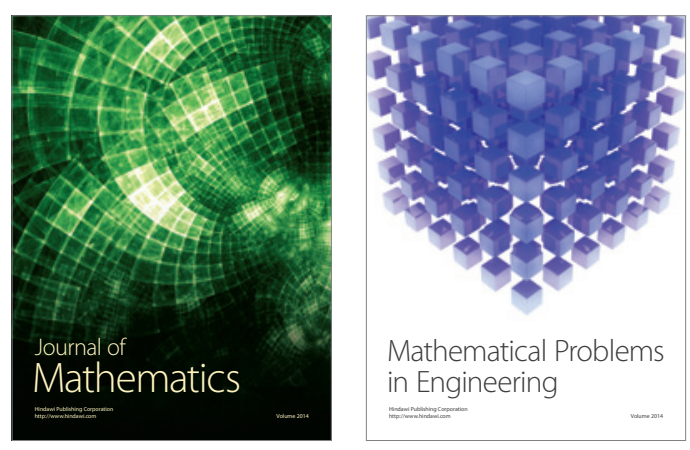

Mathematical Problems in Engineering
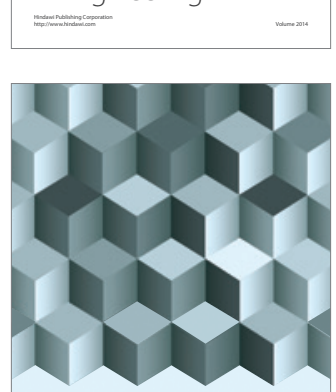

Journal of

Function Spaces
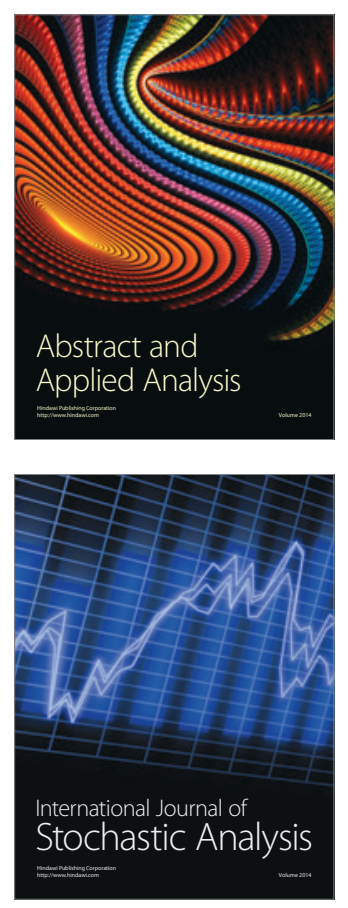

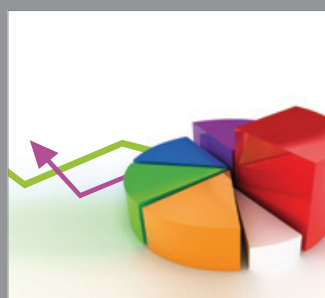

ournal of

Probability and Statistics

Promensencen
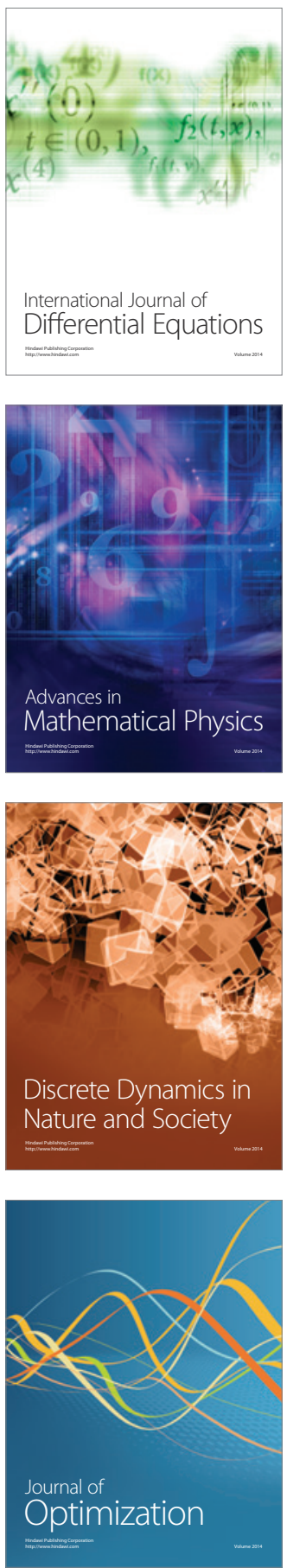